\title{
A distributed algorithm using interference pricing for relay interference channels
}

\author{
Kien T Truong and Robert W Heath, Jr
}

\begin{abstract}
Relays in cellular systems are sensitive to interference. A good relay design will transmit in a way that avoids excess out-of-cell interference. This article proposes a two-step algorithm for relay design for the relay interference channel, which models a relay-based cellular system. The transmitters and relays are equipped with multiple antennas while the receivers are equipped with a single antenna. In the first step, we propose to apply existing single-hop strategies to design the transmission parameters of the transmitters. In the second step, we propose to modify the interference pricing approach to design the relays. Interference pricing is used to provide the relays with information on how interference impacts the end-to-end achievable rates. A new method is proposed to compute interference prices via an approximation of the end-to-end achievable rate to integrate information about the relationship of the parameters in the two hops to alleviate mismatch between the rates on two hops experienced by the direct application of prior algorithms, which are designed specifically for the single-hop interference channel. Simulations show that the proposed algorithm outperforms the other designs, including the naive approach of applying the single-hop interference pricing strategies on two hops.
\end{abstract}

\section{Introduction}

The relay interference channel models multiple transmitter-receiver pairs communicating through dedicated relays using the same spectral resource [1-4], as in cellular systems with relays. On the first hop, the transmitters send data to the relays; on the second hop, after some signal processing, the relays forward data to the receivers. Each hop experiences interference, causing resource conflicts, and coupling the achievable rates for the two-hop links. In addition, the end-to-end achievable rate of a two-hop link is limited by rates achieved on each hop. Achieving high end-to-end sum-rates, therefore, requires strategies that not only mitigate interference $[4,5]$ but also match the rates on the two hops of the relay-aided links. Unfortunately, configuring the relays with limited information about the interference is challenging. Prior work on relay design for cellular systems often neglects interference [6] or ignores matching the rates in the two hops [7-9].

In this article, we maximize the end-to-end sumrates from the base stations (i.e., the transmitters) to

*Correspondence: rheath@ece.utexas.edu

Department of Electrical and Computer Engineering, The University of Texas at Austin, 1 University Station, C0806, Austin, TX 78712-0240, USA the mobile stations (i.e., the receivers) through a set of fixed relays. We assume that the transmitters send data to the receivers with the aid of fixed, half-duplex, and decode-and-forward (DF) relays via two-hop transmissions. The transmitters and relays do not share data. While the transmitters and the relays are equipped with multiple antennas, the receivers have a single antenna. The direct channels from the transmitters to the receivers are neglected due to high path-loss and shadowing attenuation. We assume that the two-hop links have a common timesharing value and perfect inter-user frame synchronization, i.e., the transmissions in each hop start at the same time and end at the same time. Although this assumption requires some coordination among the transmitters before the actual data transmission, it allows for tractable analysis to obtain insights into the system performance and provides a benchmark for the scenarios with relaxed assumptions on inter-user frame synchronization. We also assume that the parameters of the first hop are fixed and focus on the design of transmission parameters at the relays. This is reasonable when the relays are allowed to schedule users [10], as in IEEE 802.16j [11], IEEE 802.16m [12], and 3GPP LTE/LTE-Advanced standards [13]. It is important to note that we consider

\section{照 Springer}


end-to-end achievable rates. Thus, the design challenge is how to take into account information about the fixed first-hop parameters and the timesharing value in the configuration of the second hop while mitigating interference.

Our design problem is to determine transmit precoders at the transmitters and relays to maximize the end-toend sum-rates. The single-hop sum-rate maximization problem is non-convex and NP-hard, i.e., its globally optimal solution cannot be found efficiently in terms of computational complexity [14]. More complicated, the joint transmit precoder problem for end-to-end sum-rate maximization is also non-convex and is expected to be NP-hard. This motivates us to develop an algorithm for transmit precoder design that obtains high end-to-end sum-rates, i.e., to find efficiently suboptimal solutions to the end-to-end sum-rate maximization problem. Note that prior work often focuses on single-hop sum-rate maximization. Then existing algorithms could be applied to maximize independently the sum-rates in each hop of the two-hop interference channel. This naive approach, however, leads to rate mismatch in the two hops, which reduces the end-to-end sum-rates. Rate mismatch occurs when some relay links have a dominant first hop while others have a dominant second hop. From a resource allocation perspective, this means it wastes resources to the second-hop dominant relay links while depriving resources from the first-hop dominant relay links.

In this article, we propose a two-step algorithm for designing the transmit precoders at the transmitters and relays. In the first step, we propose to apply directly the existing single-hop strategies for the first hop to design the precoders at the transmitters. The relays then estimate their received signal-to-interference plus noise ratio (SINR), which represent the achievable rates on the first hop. In the second step, we use interference pricing [15-17] to develop a distributed relay beamforming algorithm where each relay determines its own transmit precoder without explicit knowledge of the precoders at the other relays and of the channels from the other relays. We propose a new method for computing interference prices at the receivers such that interference prices also include information about the first-hop SINR and the timesharing value. Our approach uses an approximation function of the end-to-end achievable rate to take into account the relationship of the parameters on two hops. We describe how the new two-hop interference pricing framework is used to develop an asynchronous-distributed pricing algorithm for relay transmit beamforming. In each iteration of the algorithm, one randomly selected relay updates its own precoder based on the knowledge of interference prices corresponding to unintended receivers by solving a nonlinear optimization problem. In general, however, finding the globally optimal solutions to this nonlinear optimization problem may be time-consuming. To overcome this challenge, we propose a method for solving it approximately, although we are unable to prove analytically the convergence of the modified algorithm. We also present in detail a version of the proposed algorithm, which we refer to as the two-hop interference pricing algorithm for relay transmit power control, where we can find closed-form expressions for the updates at the relays. This power control algorithm is applicable when the relays have a single antenna. It is also applicable when the relays have multiple antennas but we focus on designing only the norm of the relay precoders. The end-to-end sumrate performance of the proposed algorithm is evaluated by Monte Carlo simulations in a multicell cellular network. We observe that the modified algorithm converges in the simulated scenarios. For comparison, we implement a number of relay transmission strategies, including the naive application of the single-hop interference pricing algorithm in [16] to the second hop. The numerical results show that, thanks to the capabilities of interference mitigation and two-hop rate-mismatch alleviation, the proposed algorithm always outperforms all other algorithms for various system configurations.

Distributed beamforming/precoding has extensively been studied for the single-hop interference channel [16$23] ;[16,17,22]$ using interference pricing. These singlehop algorithms, including the algorithm in [16], could be applied to maximize independently the sum-rates in each hop of the two-hop interference channel. This naive approach, however, leads to rate mismatch in the two hops, which reduces the end-to-end sum-rates. On the contrary, our proposed algorithm alleviates two-hop rate mismatch by integrating the first-hop parameters and the timesharing value in relay transmit beamforming design, thus improving the end-to-end sum-rates. There have been algorithms for distributed relay beamforming [6,24-26]. Prior work, however, either focuses on another type of relay (i.e., amplify-and-forward relays [7,24,25,2729]) or considers other relay architectures (e.g., the shared relays $[6,30]$ and the two-way relay $[26,31])$. In prior work [6,24-26], each relay aids multiple pairs of transmitters and receivers at the same time. On the contrary, this study considers one-way DF relays, each of which aids only a single pair of transmitters and receivers. In principle, DF relays can be treated as conventional users on the first hop and acts as base stations on the second hop. Thus, they are readily integrated in a conventional cellular network. Amplify-and-forward relays, however, apply the linear transformation to the received signal on the first hop instead of decoding it like a conventional user. Although amplify-and-forward relays put less of a signal processing burden on the relays, their integration into conventional cellular networks requires other features like a new pilot structure and different channel estimation methods [32,33]. IEEE $802.16 \mathrm{~m}$ considered only DF relays 
[12] while 3GPP LTE/LTE-Advanced considered both DF and amplify-and-forward relays [13].

Power control is a classic technique for interference mitigation in cellular systems (see [34] and references therein). For example, there are several game theory-based power control algorithms for the single-hop interference channel [15,35-43] and femtocell networks $[44,45]$. While Huang et al. [15] uses interference pricing, Stanczak et al. $[40,43]$ use the framework of adjoint networks that allow for fully distributed implementation. Similar to the case of beamforming, since the prior algorithms are developed specifically for the single-hop interference channel, their direct applications for the second hop cause rate mismatch. The literature on power control algorithms for relay-assisted wireless networks, however, is limited [4,46-48]. An algorithm for power control at the relays and transmitters in interference relay channel is developed in [46], but it aims at minimizing the total transmit power subject to SINR requirements. The prior work in $[4,47]$ determines allocation of the sum power for each two-hop link for its transmitter and relay in a two-user two-hop interference channel to maximize the end-to-end sum-rates. Not only is the problem formulation in $[4,47]$ different from ours, but also the results cannot be easily extended to the case with more than two users. An algorithm for power control at both the base stations and relays in a multi-cell network is developed in [48]. The idea is based on the use of pricing factors to reflect the impact of interference, which is in principle similar to that of interference pricing. Nevertheless, pricing factors in [48] are determined numerically rather than by analytical methods. Also, it is unclear how the pricing factors can be extended for the beamforming design.

Our initial results in this article were reported in [49]. Compared with [49], this article presents in more detail the proposed two-hop interference pricing, discusses the distributed two-hop interference pricing algorithm, and provides more simulations that emphasize the achievable end-to-end sum-rate performance of the proposed algorithm in comparison with the existing strategies. In addition, we reported some related results on the DF relay broadcast interference channel reported in [50]. Although it also aims at the alleviating two-hop ratemismatch while mitigating interference, our prior work in [50] adopts another approach, which is based on a relationship between mean squared error (MSE) values and mutual information. Comparing the two approaches, the idea in [50] has higher channel state information (CSI) requirements and is more complex, but has the advantage of supporting multi-user multiple-input multiple-output (MIMO) from the relays.

The organization of this article is as follows. First, we introduce the system model of the relay interference channel. Second, we formulate the problem of sum end- to-end achievable rate maximization and discusses the challenges in finding its optimal solutions. Third, we present in detail the proposed approach that is based on the interference pricing framework to find high-quality suboptimal solutions. Fourth, we present Monte Carlo simulations with a multi-cell system setting. Finally, we conclude this article and provide suggestions for future research.

Notation. The lowercase and uppercase boldface letters (e.g., $\mathbf{h}$ and $\mathbf{H}$ ) indicate column vectors and matrices, respectively. $\mathbf{h}^{*}$ and $\|\mathbf{h}\|$ denote the complex conjugate transpose and the $L-2$ norm of $\mathbf{h}$. $\mathbf{I}_{N}$ stands for the identity matrix of size $N \times N$. We use $v^{\max }(\mathbf{H})$ to denote the eigenvector corresponding to the maximum eigenvalue of $\mathbf{H} .|a|$ denotes the absolute value of a complex scalar number $a$. The subscript ()$_{\{1\}}$ implies the first stage while $O_{\{2\}}$ for the second stage. The superscript ()$^{(n)}$ denotes the $n$th iteration. $\mathbb{E}[\cdot]$ is the statistical expectation operator. For any stacked notation $\mathbf{X}=\left(\mathbf{x}_{1}, \ldots, \mathbf{x}_{K}\right)$, we define $\mathbf{X}_{-k} \triangleq\left(\mathbf{x}_{1}, \ldots, \mathbf{x}_{k-1}, \mathbf{x}_{k+1}, \ldots, \mathbf{x}_{K}\right)$ for $k \in\{1, \ldots, K\}$. We use $\mathbf{X}$ and $\left(\mathbf{x}_{k} ; \mathbf{X}_{-k}\right)$ interchangeably.

\section{System model}

Consider a $K$-user relay interference channel with $K$ pairs of transmitters and receivers, as illustrated in Figure 1. We assume that the direct channels from the transmitters to the receivers are neglected due to high pathloss and shadowing attenuation. This is quite reasonable as it simply requires that the receivers in the second step not try to listen to the first hop transmission. For example, this happens in the downlink of a cellular network when the mobile stations are located near the cell boundary. One half-duplex DF relay is dedicated to each transmitter-receiver pair. A unique index $k$ from the set $\mathcal{K} \triangleq\{1, \ldots, K\}$ is assigned to each pair and its associated relay. We assume that each relay does not attempt to decode the signals of the other transmitters than its assigned one. The transmitters and relays do not share data. We assume that transmitter $k$ has $M_{k}$ antennas and relay $k$ has $N_{k}$ antennas, where $M_{k}, N_{k} \geq 1$ for $k \in \mathcal{K}$. We assume each receiver is equipped with a single antenna and focus on only the design of the relays.

Since half-duplex relays cannot transmit and receive at the same time, the transmission procedure consists of two stages. We assume the transmissions in each stage start at the same time and also end at the same time. This can be justified by some coordination between the transmitters for sharing a predefined common timesharing values and for starting sending data at the same time. The requirement is not that strict because this is typically performed as part of ranging for example in a time-division multipleaccess (TDMA) system. Such an inter-user transmission 


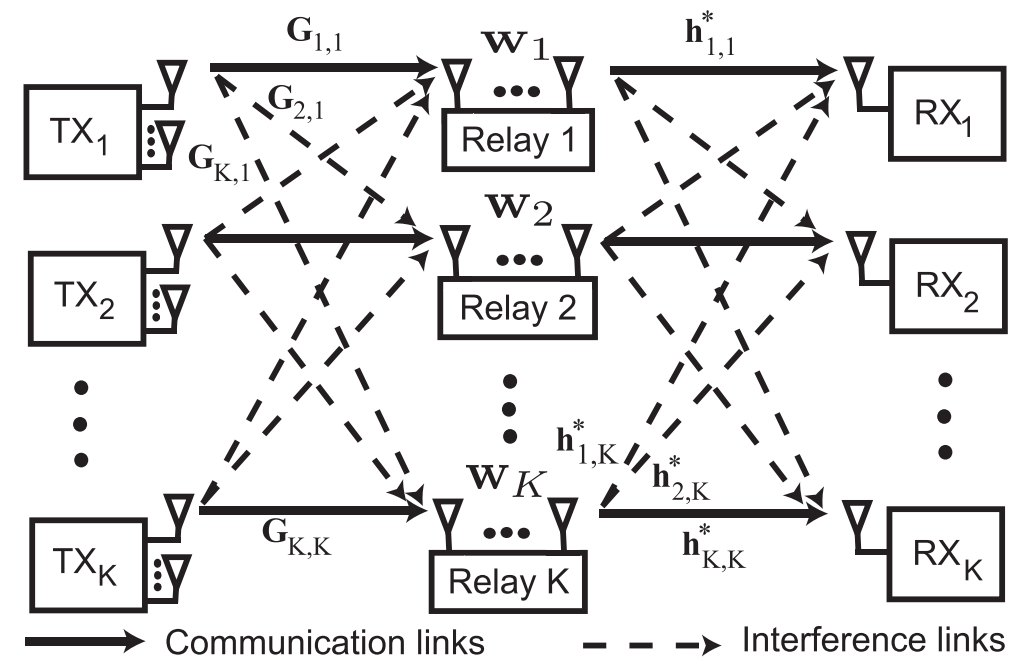

Figure 1 The $\boldsymbol{K}$-user two-hop interference channel. The transmitters (TXs) and the half-duplex DF relays are equipped with multiple antennas while the receivers (RXs) have a single antenna. Each relay aids the signal forwarding of only one TX-RX pair. The transmission procedure requires two stages. In the first stage, the TXs send data to the relays. In the second stage, each relay decodes its received signal, re-encodes and retransmits to its associated RX.

synchronization assumption is made either explicitly or implicitly in much prior work on interference channel. Moreover, this assumption allows for tractable analysis to obtain insights into the system performance and provides a benchmark for the scenarios with relaxed assumptions on inter-user frame synchronization. Using common radio resources, the transmissions in the same stage interfere with each other. For tractable analysis, we assume Gaussian signaling in both stages although it may not be optimal for the relay interference channel. In the first stage, the transmitters send data to their relays. Treating interference signals as additive Gaussian noise, each relay decodes the desired signal, re-encodes and retransmits to its receiver in the second stage. Each receiver also treats the interference from unintended relays as additive Gaussian noise when decoding the desired signal from its relay.

We consider slowly-varying, frequency-flat blockfading channels. Let $1 \leq m_{k} \leq \min \left\{M_{k}, N_{k}\right\}$ be the number of data streams that transmitter $k$ sends to relay $k$. Transmitter $k$ uses a fixed linear precoder $\mathbf{F}_{\mathrm{T}, k} \in \mathbb{C}^{M_{k} \times m_{k}}$ to map the symbol vector $\mathbf{x}_{k} \in \mathbb{C}^{m_{k} \times 1}$ to its transmit antennas. The transmitted symbols are i.i.d. such that $\mathbb{E}\left(\mathbf{x}_{k} \mathbf{x}_{k}^{*}\right)=\mathbf{I}_{m_{k}}$. Let $p_{T, k}$ be the actual transmit power and $p_{\mathrm{T}, k}^{\max }$ be the maximum transmit power at transmitter $k$. The transmit power constraint at transmitter $k$ for $k \in \mathcal{K}$ is

$$
\left\|\mathbf{F}_{\mathrm{T}, k}\right\|_{F}^{2}=p_{\mathrm{T}, k} \in \mathbb{P}_{\mathrm{T}, k} \triangleq\left[0, p_{\mathrm{T}, k}^{\max }\right] .
$$

We denote $\mathbf{G}_{k, q} \in \mathbb{C}^{N_{q} \times M_{k}}$ as the channel from transmitter $q$ to relay $k$ for $q, k \in \mathcal{K}$. Let $\mathbf{n}_{\mathrm{X}, k} \in \mathbb{C}^{N_{k} \times 1}$ denote
Gaussian noise at relay $k$ with $\mathbb{E}\left(\mathbf{n}_{\mathrm{X}, k} \mathbf{n}_{\mathrm{X}, k}^{*}\right)=\sigma_{\mathrm{X}, k}^{2} \mathbf{I}_{N_{\mathrm{X}, k}}$. We define $\mathbf{F}_{\mathrm{T}} \triangleq\left(\mathbf{F}_{\mathrm{T}, 1}, \ldots, \mathbf{F}_{\mathrm{T}, K}\right) \in \mathbb{F}_{\mathrm{T}} \triangleq \mathbb{C}^{M_{1} \times m_{1} \times}$ $\cdots \times \mathbb{C}^{M_{K} \times m_{K}}$ and $\mathbf{p}_{\mathrm{T}} \triangleq\left(p_{\mathrm{T}, 1}, \ldots, p_{\mathrm{T}, K}\right) \in \mathbb{P}_{\mathrm{T}} \triangleq \mathbb{P}_{\mathrm{T}, 1} \times$ $\cdots \times \mathbb{P}_{\mathrm{T}, K}$. Relay $k$ observes

$$
\mathbf{y}_{\mathrm{X}, k}=\mathbf{G}_{k, k} \mathbf{F}_{\mathrm{T}, k} \mathbf{x}_{k}+\sum_{q \neq k} \mathbf{G}_{k, q} \mathbf{F}_{\mathrm{T}, q} \mathbf{x}_{q}+\mathbf{n}_{\mathrm{X}, k} .
$$

The interference plus noise covariance matrix at relay $k$ is

$$
\mathbf{R}_{\mathrm{X}, k}\left(\mathbf{F}_{\mathrm{T}}\right)=\sum_{q \neq k} \mathbf{G}_{k, q} \mathbf{F}_{\mathrm{T}, q} \mathbf{F}_{\mathrm{T}, q}^{*} \mathbf{G}_{k, q}^{*}+\sigma_{\mathrm{X}, k}^{2} \mathbf{I}_{N_{\mathrm{X}, k}} .
$$

The maximum achievable rate from transmitter $k$ to relay $k$ is

$R_{1, k}\left(\mathbf{F}_{\mathrm{T}}\right)=\log _{2} \operatorname{det}\left(\mathbf{I}_{N}+\mathbf{F}_{\mathrm{T}, k}^{*} \mathbf{G}_{k, k}^{*}\left[\mathbf{R}_{\mathrm{X}, k}\left(\mathbf{F}_{\mathrm{T}}\right)\right]^{-1} \mathbf{G}_{k, k} \mathbf{F}_{\mathrm{T}, k}\right)$.

Define $\xi_{k}\left(\mathbf{F}_{\mathrm{T}}\right)=2^{R_{1, k}\left(\mathbf{F}_{\mathrm{T}}\right)}-1$. Intuitively, one can think of $\xi_{k}\left(\mathbf{F}_{\mathrm{T}}\right)$ as an effective SINR at relay $k$ if transmitter $k$ sends a single data stream to relay $k$.

Since relay $k$ is dedicated to aiding the $k$ th pair by assumption, it attempts to decode only $\mathbf{x}_{k}$. After that, relay $k$ re-encodes the signal as $r_{k}$ such that $\mathbb{E}\left(\left|r_{k}\right|^{2}\right)=1$. Relay $k$ uses a linear beamforming vector $\mathbf{f}_{\mathrm{X}, k} \in \mathbb{C}^{N_{k} \times 1}$ to transmit $r_{k}$ to receiver $k$. Let $p_{\mathrm{X}, k}$ be the actual transmit power and $p_{\mathrm{X}, k}^{\max }$ be the maximum transmit power at relay $k$. The transmit power constraint at relay $k$ for $k \in \mathcal{K}$ is

$$
\left\|\mathbf{f}_{\mathrm{X}, k}\right\|_{F}^{2}=p_{\mathrm{X}, k} \in \mathbb{P}_{\mathrm{X}, k} \triangleq\left[0, p_{\mathrm{X}, k}^{\max }\right] .
$$

We define $\mathbf{F}_{\mathrm{X}} \triangleq\left(\mathbf{f}_{\mathrm{X}, 1}, \ldots, \mathbf{f}_{\mathrm{X}, K}\right) \in \mathbb{F}_{\mathrm{X}} \triangleq \mathbb{C}^{N_{1} \times 1} \times \cdots \times$ $\mathbb{C}^{N_{K} \times 1}$ and $\mathbf{P}_{\mathrm{X}} \triangleq\left(p_{\mathrm{X}, 1} \ldots p_{\mathrm{X}, K}\right) \in \mathbb{P}_{\mathrm{X}, 1} \times \cdots \times \mathbb{P}_{\mathrm{X}, K}$. 
Let $\mathbf{h}_{k, m}^{*}$ denote the channel from relay $m$ to receiver $k$, where $\mathbf{h}_{k, m} \in \mathbb{C}^{N_{k} \times 1}$. We denote $n_{\mathrm{R}, k}$ as the additive spatially white Gaussian noise at receiver $k$ for $k \in \mathcal{K}$ with variance $\sigma_{\mathrm{R}, k}^{2}$. Receiver $k$ observes

$$
y_{k}=\mathbf{h}_{k, k}^{*} \mathbf{f}_{\mathrm{X}, k} r_{k}+\sum_{q \neq k} \mathbf{h}_{k, q}^{*} \mathbf{f}_{\mathrm{X}, q} r_{q}+n_{\mathrm{R}, k} .
$$

Define the signal power as $A_{k}\left(\mathbf{F}_{\mathrm{X}}\right) \triangleq \mathbf{f}_{\mathrm{X}, k}^{*} \mathbf{h}_{k, k} \mathbf{h}_{k, k}^{*} \mathbf{f}_{\mathrm{X}, k}$ and the sum interference power as $I_{k}\left(\mathbf{F}_{\mathrm{X}}\right) \triangleq \sum_{q \neq k} \mathbf{f}_{\mathrm{X}, q}^{*}$ $\mathbf{h}_{k, q} \mathbf{h}_{k, q}^{*} \mathbf{f}_{\mathrm{X}, q}$. The SINR at receiver $k$ is

$$
\gamma_{k}\left(\mathbf{F}_{\mathrm{X}}\right)=\frac{A_{k}\left(\mathbf{F}_{\mathrm{X}}\right)}{I_{k}\left(\mathbf{F}_{\mathrm{X}}\right)+\sigma_{\mathrm{R}, k}^{2}} .
$$

The maximum achievable rate of the $k$ th second-hop link is given by

$$
R_{2, k}\left(\gamma_{k}\left(\mathbf{F}_{\mathrm{X}}\right)\right)=\log _{2}\left(1+\gamma_{k}\left(\mathbf{F}_{\mathrm{X}}\right)\right) .
$$

Let $t \in(0,1)$ be the fraction of time allocated for the first stage, which is also referred to as the timesharing value. The fraction of time for the second stage is $(1-t)$. For example, in 3GPP LTE/LTE-Advanced cellular systems, $t$ depends on the number of subframes for backhaul links (i.e., between base stations and relays) in a radio frame [13]. We assume that $t$ is a given parameter. Finding the optimal value for $t$ is an interesting problem but it increases the requirements for synchronization and coordination among the two-hop links. The normalized rate of the $k$ th first-hop link is $t R_{1, k}\left(\mathbf{F}_{\mathrm{T}}\right)$ while the normalized rate of the $k$ th second-hop link is $(1-t) R_{2, k}\left(\mathbf{F}_{\mathrm{X}}\right)$. The end-toend achievable rate of the link from transmitter $k$ via relay $k$ to receiver $k$ is defined as the smaller of the normalized rates on two hops [51]

$$
R_{k}\left(\mathbf{F}_{\mathrm{T}}, \mathbf{F}_{\mathrm{X}}\right)=\min \left(t R_{1, k}\left(\mathbf{F}_{\mathrm{T}}\right),(1-t) R_{2, k}\left(\mathbf{F}_{\mathrm{X}}\right)\right) .
$$

The end-to-end sum-rates is defined as

$$
R_{\text {sum }}\left(\mathbf{F}_{\mathrm{T}}, \mathbf{F}_{\mathrm{X}}\right) \triangleq \sum_{k=1}^{K} R_{k}\left(\mathbf{F}_{\mathrm{T}}, \mathbf{F}_{\mathrm{X}}\right) .
$$

The design of $\mathbf{F}_{\mathrm{T}}$ and $\mathbf{F}_{\mathrm{X}}$ for maximizing $R_{\text {sum }}\left(\mathbf{F}_{\mathrm{T}}, \mathbf{F}_{\mathrm{X}}\right)$ should take into account $t$. Also, note that the units for the achievable rates in (3), (6), (7), and (8) are bps/Hz.

\section{Problem formulation}

The joint precoder design and power control problem for end-to-end sum-rate maximization in the DF relay interference channel is formulated as

$$
(\mathcal{O P}): \max _{\left(\mathbf{F}_{\mathrm{T}}, \mathbf{F}_{\mathrm{X}}\right) \in \mathbb{F}_{\mathrm{T}} \times \mathbb{F}_{\mathrm{X}}} R_{\text {sum }}\left(\mathbf{F}_{\mathrm{T}}, \mathbf{F}_{\mathrm{X}}\right) .
$$

Interference mitigation is the main challenge in sum-rate maximization. Due to interference, there exists coupling among the achievable rates on the same hop. It is the coupling that makes sum-rate maximization problems nonconvex and NP-hard, even for the single-hop interference channel $[52,53]$. The more complicated problem $(\mathcal{O P})$ is expected to be non-convex and NP-hard as well. This means that the globally optimal solutions to $(\mathcal{O P})$ cannot be found efficiently in terms of computational complexity even in a centralized fashion. In addition, due to the definition of the end-to-end achievable rates, $(\mathcal{O P})$ has a complicated per-user objective function. In fact, it is challenging to find its stationary points, including their globally and locally optimal solutions [54]. Thus, in this article, we focus on finding suboptimal solutions to $(\mathcal{O P})$ that have high end-to-end sum-rates.

Note that two-hop rate matching is a challenge in solving for suboptimal solutions to $(\mathcal{O P})$. Specifically, for a given $t, \mathbf{F}_{\mathrm{T}}$ and $\mathbf{F}_{\mathrm{X}}$, there may exist a mismatch between the normalized rates on two hops. By definition, a twohop rate mismatch occurs when there exist two two-hop links such that one has the dominant first-hop link while the other has the dominant second-hop link. Mathematically, it occurs when there exist $k, m \in \mathcal{K}_{\mathrm{X}}$ and $k \neq m$, such that $t R_{1, k}\left(\mathbf{F}_{\mathrm{T}}\right)>(1-t) R_{2, k}\left(\mathbf{F}_{\mathrm{X}}\right)$ and $t R_{1, m}\left(\mathbf{F}_{\mathrm{T}}\right)<$ $(1-t) R_{2, m}\left(\mathbf{F}_{\mathrm{X}}\right)$. When a two-hop rate mismatch happens, we can always improve the end-to-end sum-rates by scaling down $p_{\mathrm{X}, m}$ and fixing all the other parameters such that the rates on two hops of the $m$ th two-hop link are equal to $t R_{1, m}\left(\mathbf{F}_{\mathrm{T}}\right)$. While this power reduction does not change $R_{m}\left(\mathbf{F}_{\mathrm{T}}, \mathbf{F}_{\mathrm{X}}\right)$, it decreases the interference caused by relay $m$ to unintended receivers on the second hop. This means that the power reduction does not decrease $R_{q}\left(\mathbf{F}_{\mathrm{T}}, \mathbf{F}_{\mathrm{X}}\right)$ for $q \neq m$, and especially it strictly increases $R_{k}\left(\mathbf{F}_{\mathrm{T}}, \mathbf{F}_{\mathrm{X}}\right)$. Thus, an efficient system design in terms of end-to-end sum-rate maximization should not cause any two-hop rate mismatch.

\section{A distributed algorithm using interference pricing}

In this section, we propose a distributed algorithm for finding high-quality suboptimal solutions to $(\mathcal{O P})$. The proposed algorithm consists of two consecutive steps: (i) the first step focuses on designing the transmitters while ignoring the second-hop parameters and (ii) the second step focuses on designing the relays given knowledge of the timesharing value and the first-hop achievable rates resulting from the previous step.

\section{Step 1: transmitter design}

In the first step, we focus only on the design of the parameters of the transmitters on the first hop. In particular, we use one of the existing distributed algorithms for the single-hop interference channel to design the first-hop precoders $\mathbf{F}_{\mathrm{T}}$ and/or transmit power values $\mathbf{p}_{\mathrm{T}}$. Due to two-hop rate mismatch, an algorithm with a lower first-hop sum-rates may achieve higher end-to-end sum-rates than another algorithm with a higher firsthop sum-rates. Thus, in our proposed two-step algorithm, all the existing distributed transmission strategies for the 
single-hop interference channel are candidates for designing the transmitters' parameters.

We now briefly describe several strategies for the design of multiple-antenna transmitters with increasing requirements for complexity and overhead:

- In the first strategy, each transmitter aims at maximizing the desired signal to its associated relay regardless of the interference it may cause to unintended relays [55]. This strategy has the lowest complexity and overhead and is referred to as the maximum ratio transmission (MRT) beamforming. From a game theoretic perspective, the transmitters behave egoistically in this strategy, resulting in no cooperation among them [56]. Each transmitter is required to know only the channel to its associated relay, thus allowing for completely distributed implementation.

- In the second strategy, each transmitter behaves altruistically by minimizing the power of interference they cause to unintended relays. The interference on the transmit side is also known as the leakage $[21,57,58]$. Specifically, each transmitter uses multiple-antenna techniques for nullifying its leakage signals, similar to zero-forcing (ZF) beamforming on the receive side. The implementation of the strategy requires that each transmitter has the CSI of the channels from itself to all the relays. Thus, it also allows for distributed implementation but with a larger amount of overhead than the MRT strategy.

- In the third strategy, each transmitter aims at maximizing the signal-to-leakage plus noise ratio (SLNR), which is also known as the virtual SINR [59]. The SLNR for each transmitter is defined as the ratio of the desired signal power at its receiver over the sum power of its leakage signals. The maximization of the SLNR provides some balance between egoism and altruism. Note that, in addition to the CSI of the channel from itself to all the relays, each transmitter needs to know the variance of the noise at its associated receiver.

- In the fourth strategy, each transmitter determines its own beamforming vector based on knowledge of how it affects the achievable rates of unintended receivers. This is based on the interference pricing framework, which was developed for the single-hop interference channel in $[15-17,35,60]$. When applied to the first hop, the relays compute interference prices that quantify marginal changes in the achievable rates per unit increase in interference power at the relays [15]. The relays then provide their interference prices to the transmitters via feedback channels. Using the interference prices and the knowledge of the channels from itself to the relays, each transmitter determines its own beamforming vector. Although this strategy allows for distributed implementation, it is iterative and requires more overhead due to the exchange of interference prices.

- In the final strategy, each transmitter aims at minimizing its corresponding weighted MSE. The idea is to formulate a weighted MSE minimization problem that has the same stationary points as the sum-rate maximization problem but has a better-behaved objective function based on a relationship between the mutual information and MSE [61]. Thus, we can solve for the stationary points of the weighted MSE minimization problem instead of finding directly those of the sum-rate maximization problem. Note that our other results in [50] are based on the same approach as this strategy.

Let $\bar{\xi}_{k}$ be the resulting received SINR at relay $k$ for $k \in \mathcal{K}$ at the end of the first step. Recall that, by definition, these values represent the achievable rates on the first hop and are used as an input for the relay design in the second step. We assume that relay $k$ knows $\bar{\xi}_{k}$ perfectly and use it in the relay design. In principle, we can try several candidate distributed strategies in designing the transmitters in the first step and then provide the corresponding values of $\left\{\bar{\xi}_{k}\right\}_{k=1}^{K}$ for the relay design. The relays will be designed with different sets of $\left\{\bar{\xi}_{k}\right\}_{k=1}^{K}$ to select the one with the highest end-to-end sum-rates. The selected candidate first-hop design strategy will then be informed to the transmitters. Although improving the end-to-end sum-rates, this increases the requirements for coordination and overhead.

\section{Step 2: relay design}

Given the transmitter design in the first step, we can rewrite the end-to-end rate of the $k$ th two-hop link as

$$
R_{k}\left(\bar{\xi}_{k}, \gamma_{k}\left(\mathbf{F}_{X}\right)\right)=\min \left\{t \log _{2}\left(1+\bar{\xi}_{k}\right),(1-t) \log _{2}\left(1+\gamma_{k}\left(\mathbf{F}_{\mathrm{X}}\right)\right)\right. \text {. }
$$

The design problem now becomes

$$
(\mathcal{B F}): \max _{\left(\mathbf{F}_{\mathrm{X}}\right) \in \mathbb{F}_{\mathrm{X}}} R_{k}\left(\bar{\xi}_{k}, \gamma_{k}\left(\mathbf{F}_{\mathrm{X}}\right)\right) \text {. }
$$

By definition, the end-to-end sum-rates depend not only on the second-hop achievable rates, but also on $t$ and $\left\{\bar{\xi}_{k}\right\}_{k=1}^{K}$. Thus, in addition to mitigating interference to achieve high second-hop rates, the relay design should match the rates achieved on the second hop with those achieved on the first hop as designed in the previous step. In the second step, given the transmitters' parameters designed in the first step, we adopt an interference pricing approach to design the relays' parameters for obtaining high end-to-end sum-rates. The key idea is to take the 
advantage of interference prices to exchange information about $t$ and $\left\{\bar{\xi}_{k}\right\}_{k=1}^{K}$ between the relays and receivers for a better relay design. One of our main contributions is to propose a modification of the computation method of interference prices to integrate information about $t$ and $\left\{\bar{\xi}_{k}\right\}_{k=1}^{K}$, which we refer to as the two-hop interference pricing framework. We also apply the new framework to develop the corresponding algorithm for solving $(\mathcal{B F})$.

\section{Proposed two-hop interference pricing framework}

We now present the two-hop interference pricing framework for transmit beamforming. Note that it is straightforward to develop the similar two-hop interference pricing framework for power control, however, we omit it here to save space. The interference prices are computed based on the Karush-Kuhn-Tucker (KKT) necessary conditions for optimality of the sum-rate maximization problem. This computation method is crucial for interference pricing-based algorithms to obtain high sum-rates. In the DF relay interference channel, we notice that $R_{k}\left(\bar{\xi}_{k}, \gamma_{k}\left(\mathbf{F}_{\mathrm{X}}\right)\right)$ is not continuously differentiable with respect to $\gamma_{k}\left(\mathbf{F}_{X}\right)$ at the intersection point that makes $(1-t) \log _{2}\left(1+\gamma_{k}\left(\mathbf{F}_{\mathrm{X}}\right)\right)$ equal to $t \log _{2}\left(1+\bar{\xi}_{k}\right)$. It follows that $R_{k}\left(\bar{\xi}_{k}, \gamma_{k}\left(\mathbf{F}_{\mathrm{X}}\right)\right)$ is not continuously differentiable with respect to $\mathbf{f}_{\mathrm{X}, m}$ at every point for all $m \in \mathcal{K}$. Therefore, it is challenging to use the KKT necessary conditions for $(\mathcal{B F})$ to find directly even its locally optimal solutions to [62]. To overcome this, we propose to use an approximate function of the end-to-end achievable rate, which we refer to as $R_{\text {soft }, k}\left(\gamma_{k}\left(\mathbf{F}_{\mathrm{X}}\right)\right)$ and is a function of $\left\{\bar{\xi}_{k}\right\}_{k=1}^{K}, t$, and $\gamma_{k}\left(\mathbf{F}_{\mathrm{X}}\right)$.

Some guidelines for selecting $R_{\mathrm{soft}, k}\left(\gamma_{k}\left(\mathbf{F}_{\mathrm{X}}\right)\right)$ are provided. One criterion is that $R_{\mathrm{soft}, k}\left(\gamma_{k}\left(\mathbf{F}_{\mathrm{X}}\right)\right)$ is continuously differentiable with respect to $\gamma_{k}\left(\mathbf{F}_{\mathrm{X}}\right)$ at every point to make it possible to use the KKT conditions. Another criterion is that the utility function for each user has an appropriate concavity so that the convergence of the resulting interference pricing algorithm is guaranteed [35]. Specifically, $R_{\text {soft }, k}\left(\gamma_{k}\left(\mathbf{F}_{\mathrm{X}}\right)\right)$ has to satisfy the following condition [35]

$$
C_{k}\left(R_{\mathrm{soft}, k}\left(\gamma_{k}\left(\mathbf{F}_{\mathrm{X}}\right)\right)\right) \triangleq-\frac{R_{\mathrm{soft}, k}^{\prime \prime}\left(\gamma_{k}\left(\mathbf{F}_{\mathrm{X}}\right)\right) \gamma_{k}\left(\mathbf{F}_{\mathrm{X}}\right)}{R_{\mathrm{soft}, k}^{\prime}\left(\gamma_{k}\left(\mathbf{F}_{\mathrm{X}}\right)\right)} \in(0,2] .
$$

The quantity $C_{k}\left(R_{\text {soft }, k}\left(\gamma_{k}\left(\mathbf{F}_{\mathrm{X}}\right)\right)\right)$ is referred to as the coefficient of relative risk aversion of the utility function $R_{\text {soft }, k}\left(\gamma_{k}\left(\mathbf{F}_{\mathrm{X}}\right)\right)$ [63]. The larger value of $C_{k}\left(R_{\mathrm{soft}, k}\left(\gamma_{k}\left(\mathbf{F}_{\mathrm{X}}\right)\right)\right)$ indicates that $R_{\text {soft }, k}\left(\gamma_{k}\left(\mathbf{F}_{\mathrm{X}}\right)\right)$ is "more concave" [35]. If these conditions are satisfied, then it is possible to develop an algorithm with asynchronous beamforming vector updates such that $\sum_{k=1}^{K} R_{\text {soft }, k}\left(\gamma_{k}\left(\mathbf{F}_{\mathrm{X}}\right)\right)$ is non-decreasing after each iteration provided that the interference prices are current (i.e., they are updated after each iteration) $[16,35]$.
Define $\phi_{k}\left(\bar{\xi}_{k}, t\right)$ as the rate-matching received SINR for the $k$ th second-hop link, which is the value of the received SINR at receiver $k$ if the normalized rates on two hops are equal to each other. Specifically, by setting $t \log _{2}\left(1+\bar{\xi}_{k}\right)=$ $(1-t) \log _{2}\left(1+\phi_{k}\left(\bar{\xi}_{k}, t\right)\right)$ and after some manipulation we obtain

$$
\phi_{k}\left(\bar{\xi}_{k}, t\right)=\left(1+\bar{\xi}_{k}\right)^{\frac{t}{1-t}}-1
$$

Recall that one challenge is that $R_{k}\left(\bar{\xi}_{k}, \gamma_{k}\left(\mathbf{F}_{\mathbf{X}}\right)\right)$ is not continuously differentiable with respect to $\gamma_{k}\left(\mathbf{F}_{X}\right)$ at the point $\gamma_{k}\left(\mathbf{F}_{X}\right)=\phi_{k}\left(\bar{\xi}_{k}, t\right)$. We propose a method to find a class of approximate functions that are continuously differentiable at every point and are exactly equal to $R_{k}\left(\bar{\xi}_{k}, \gamma_{k}\left(\mathbf{F}_{\mathrm{X}}\right)\right)$ when $\gamma_{k}\left(\mathbf{F}_{\mathrm{X}}\right) \leq \phi_{k}\left(\bar{\xi}_{k}, t\right)$ as shown in Figure 2. Using this method, we now provide the following two approximate functions $R_{\mathrm{ip} 1, k}\left(\gamma_{k}\left(\mathbf{F}_{\mathrm{X}}\right)\right)$ and $R_{\mathrm{ip} 2, k}\left(\gamma_{k}\left(\mathbf{F}_{\mathrm{X}}\right)\right)$ in that class

$$
\begin{aligned}
& R_{\mathrm{ip} 1, k}\left(\gamma_{k}\left(\mathbf{F}_{\mathrm{X}}\right)\right) \\
& = \begin{cases}(1-t) \log _{2}\left(1+\gamma_{k}\left(\mathbf{F}_{\mathrm{X}}\right)\right), & \text { if } \gamma_{k}\left(\mathbf{F}_{\mathrm{X}}\right) \leq \phi_{k}\left(\bar{\xi}_{k}, t\right) \\
\log _{2}\left(1+\bar{\xi}_{k}\right) & \\
\quad+\frac{1-t}{\ln 2} \frac{\gamma_{k}\left(\mathbf{F}_{\mathrm{X}}\right)-\phi_{k}\left(\bar{\xi}_{k}, t\right)}{1+\gamma_{k}\left(\mathbf{F}_{\mathrm{X}}\right)}, & \text { otherwise, }\end{cases}
\end{aligned}
$$

$$
\begin{aligned}
& R_{\text {ip } 2, k}\left(\gamma_{k}\left(\mathbf{F}_{\mathrm{X}}\right)\right) \\
& =\left\{\begin{array}{l}
(1-t) \log _{2}\left(1+\gamma_{k}\left(\mathbf{F}_{\mathrm{X}}\right)\right), \\
t \log _{2}\left(1+\bar{\xi}_{k}\right)+\frac{1-t}{\ln 2} \frac{\phi_{k}\left(\bar{\xi}_{k}, t\right)}{2} \\
\times\left[1-\exp \left(\frac{2\left(\phi_{k}\left(\bar{\xi}_{k}, t\right)-\gamma_{k}\left(\mathbf{F}_{\mathrm{X}}\right)\right)}{\left[\gamma_{k}\left(\mathbf{F}_{\mathrm{X}}\right)+1\right]\left[\phi_{k}\left(\bar{\xi}_{k}, t\right)+1\right]}\right)\right], \text { otherwise. }
\end{array}\right.
\end{aligned}
$$

The coefficients of relative risk aversion of the approximate functions are given by

$$
\begin{aligned}
& C_{k}\left(R_{\mathrm{ip} 1, k}\left(\gamma_{k}\left(\mathbf{F}_{\mathrm{X}}\right)\right)\right) \\
& = \begin{cases}\frac{\gamma_{k}\left(\mathbf{F}_{\mathrm{X}}\right)}{\left(1+\gamma_{k}\left(\mathbf{F}_{\mathrm{X}}\right)\right)}, & \text { if } \gamma_{k}\left(\mathbf{F}_{\mathrm{X}}\right) \leq \phi_{k}\left(\bar{\xi}_{k}, t\right) \\
\frac{2 \gamma_{k}\left(\mathbf{F}_{\mathrm{X}}\right)}{\left(1+\gamma_{k}\left(\mathbf{F}_{\mathrm{X}}\right)\right)}, & \text { otherwise, }\end{cases} \\
& C_{k}\left(R_{\mathrm{ip} 2, k}\left(\gamma_{k}\left(\mathbf{F}_{\mathrm{X}}\right)\right)\right) \\
& = \begin{cases}\frac{\gamma_{k}\left(\mathbf{F}_{\mathrm{X}}\right)}{\left(1+\gamma_{k}\left(\mathbf{F}_{\mathrm{X}}\right)\right)}, & \text { if } \gamma_{k}\left(\mathbf{F}_{\mathrm{X}}\right) \leq \phi_{k}\left(\bar{\xi}_{k}, t\right) \\
\frac{2 \gamma_{k}\left(\mathbf{F}_{\mathrm{X}}\right)\left(2+\gamma_{k}\left(\mathbf{F}_{\mathrm{X}}\right)\right)}{\left(1+\gamma_{k}\left(\mathbf{F}_{\mathrm{X}}\right)\right)^{2}}, & \text { otherwise. }\end{cases}
\end{aligned}
$$




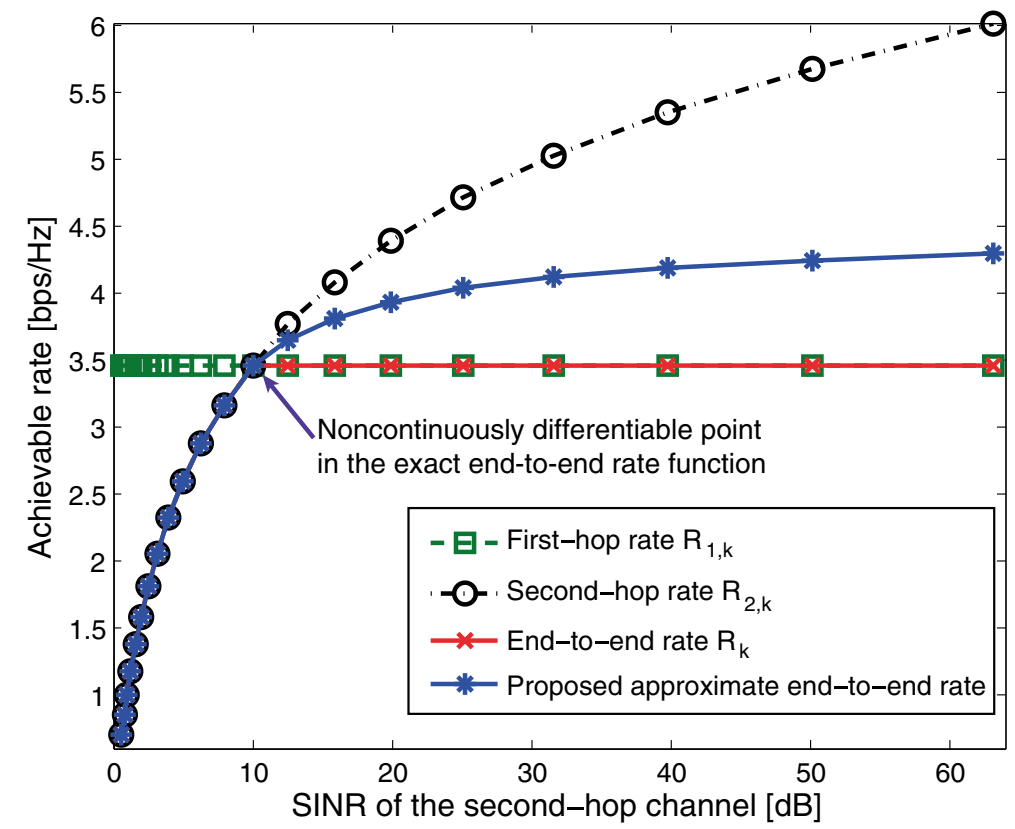

Figure 2 Illustration of how we find approximation functions. The achievable rates related to the kth two-hop link as well as the proposed approximate end-to-end achievable rate for the two-hop interference pricing framework are plotted as functions of the SINR of the second-hop link. The SINR of the first-hop link is $10 \mathrm{~dB}$.

Because $0<\gamma_{k}\left(\mathbf{F}_{\mathrm{X}}\right)<\left(1+\gamma_{k}\left(\mathbf{F}_{\mathrm{X}}\right)\right)$ and $0<\gamma_{k}(2+$ $\left.\gamma_{k}\left(\mathbf{F}_{\mathrm{X}}\right)\right)<\left(1+\gamma_{k}\left(\mathbf{F}_{\mathrm{X}}\right)\right)^{2}$, we have $C_{k}\left(R_{\mathrm{ip} 1, k}\left(\gamma_{k}\left(\mathbf{F}_{\mathrm{X}}\right)\right)\right)$, $C_{k}\left(R_{\mathrm{ip} 2, k}\left(\gamma_{k}\left(\mathbf{F}_{\mathrm{X}}\right)\right)\right) \in(0,2]$. Both $R_{\mathrm{ip} 1, k}\left(\gamma_{k}\left(\mathbf{F}_{\mathrm{X}}\right)\right)$ and $R_{\mathrm{ip} 2, k}\left(\gamma_{k}\left(\mathbf{F}_{\mathrm{X}}\right)\right)$ are functions of $\gamma_{k}\left(\mathbf{F}_{\mathrm{X}}\right), t$ and $\left\{\bar{\xi}_{k}\right\}_{k=1}^{K}$. If $\gamma_{k}\left(\mathbf{F}_{X}\right)>\phi_{k}\left(\bar{\xi}_{k}, t\right)$, then they are larger than $R_{k}\left(\bar{\xi}_{k}, \gamma_{k}\left(\mathbf{F}_{\mathrm{X}}\right)\right)$. The gaps between these approximate functions and the exact function increase with $\gamma_{k}\left(\mathbf{F}_{X}\right)$ and are upperbounded by $(1-t) / \ln 2$. It is still unclear how to evaluate the quality of the approximate endto-end functions in terms of exact end-to-end sumrate maximization. Roughly speaking, we expect that the one with a smaller gap with the exact function, which is $R_{\mathrm{ip} 2, k}\left(\gamma_{k}\left(\mathbf{F}_{\mathrm{X}}\right)\right)$ in this case, outperforms the other. Although this prediction is confirmed by our numerical results, we do not have a formal proof. Note that the two approximate functions can be used to develop distributed two-hop interference pricing algorithms for solving $(\mathcal{B F})$ and $(\mathcal{P C})$. The next sections present the details of how such algorithms are developed using $R_{\mathrm{ip} 1, k}\left(\gamma_{k}\left(\mathbf{F}_{\mathrm{X}}\right)\right)$. The algorithms corresponding to the use of $R_{\mathrm{ip} 2, k}\left(\gamma_{k}\left(\mathbf{F}_{\mathrm{X}}, \mathbf{p}_{\mathrm{X}}\right)\right)$ can be developed in the same way.

\section{Distributed algorithm development}

Let $(\mathcal{B F}-\mathcal{I P})$ be the relay beamforming design problem for approximate end-to-end sum-rate maximization associated with $R_{\mathrm{ip} 1, k}\left(\gamma_{k}\left(\mathbf{F}_{\mathrm{X}}\right)\right)$. This can be formulated as

$$
(\mathcal{B F}-\mathcal{I P}): \max _{\mathbf{F}_{\mathrm{X}} \in \mathbb{F}_{\mathrm{X}}} \sum_{k=1}^{K} R_{\mathrm{ip} 1, k}\left(\gamma_{k}\left(\mathbf{F}_{\mathrm{X}}\right)\right)
$$

This section describes a distributed two-hop interference pricing algorithm for solving $(\mathcal{B F}-\mathcal{I P})$.

We first present the computation method of interference prices. Consider a set of dual variables $\lambda_{1}, \ldots, \lambda_{K} \geq 0$ the constraints $\left\|\mathbf{f}_{\mathrm{X}, k}\right\|^{2} \leq p_{\mathrm{X}, k}^{\max }$ for $k \in \mathcal{K}$. The KKT conditions for $(\mathcal{B F}-\mathcal{I P})$ are

$$
\begin{aligned}
& \frac{\partial R_{\mathrm{ip} 1, k}\left(\gamma_{k}\left(\mathbf{F}_{\mathrm{X}}\right)\right)}{\partial \mathbf{f}_{\mathrm{X}, k}^{*}}+\sum_{m \neq k} \frac{\partial R_{\mathrm{ip} 1, m}\left(\gamma_{m}\left(\mathbf{F}_{\mathrm{X}}\right)\right)}{\partial \mathbf{f}_{\mathrm{X}, k}^{*}}=\lambda_{k} \mathbf{f}_{\mathrm{X}, k}, \\
& \lambda_{k}\left(\left\|\mathbf{f}_{\mathrm{X}, k}\right\|^{2}-p_{\mathrm{X}, k}^{\max }\right)=0 .
\end{aligned}
$$

Recall that for the $k$ th second-hop link, we define the signal power as $A_{k}\left(\mathbf{F}_{\mathrm{X}}\right)=\mathbf{f}_{\mathrm{X}, k}^{*} \mathbf{h}_{k, k} \mathbf{h}_{k, k}^{*} \mathbf{f}_{\mathrm{X}, k}$ and the sum interference power as $I_{k}\left(\mathbf{F}_{\mathrm{X}}\right)=\sum_{q \neq k} \mathbf{f}_{\mathrm{X}, q}^{*} \mathbf{h}_{k, q} \mathbf{h}_{k, q}^{*} \mathbf{f}_{\mathrm{X}, q}$. Define the following values for all $k \in \mathcal{K}$

$$
\begin{aligned}
& \theta_{\mathrm{BF}, \mathrm{ip} 1, k}\left(\mathbf{F}_{\mathrm{X}}\right)=-\frac{\partial R_{\mathrm{ip} 1, k}\left(\gamma_{k}\left(\mathbf{F}_{\mathrm{X}}\right)\right)}{\partial I_{k}\left(\mathbf{F}_{\mathrm{X}}\right)}, \\
& \beta_{\mathrm{BF}, \mathrm{ip} 1, k}\left(\mathbf{F}_{\mathrm{X}}\right)=\frac{\partial R_{\mathrm{ip} 1, k}\left(\gamma_{k}\left(\mathbf{F}_{\mathrm{X}}\right)\right)}{\partial A_{k}\left(\mathbf{F}_{\mathrm{X}}\right)} .
\end{aligned}
$$

Note that $\theta_{\mathrm{BF}, \mathrm{ip} 1, k}\left(\mathbf{F}_{\mathrm{X}}\right)$ represents the marginal decrease in $R_{\mathrm{ip} 1, k}\left(\gamma_{k}\left(\mathbf{F}_{\mathrm{X}}\right)\right)$ per unit increase in $I_{k}\left(\mathbf{F}_{\mathrm{X}}\right)$. Thus, we can interpret $\theta_{\mathrm{BF}, \mathrm{ip} 1, k}\left(\mathbf{F}_{\mathrm{X}}\right)$ as the cost charged to any relay $m \neq k$ for generating interference to receiver $k$ or as the 
interference price at receiver $k$. Similarly, $\beta_{\mathrm{BF}, \mathrm{ip} 1, k}\left(\mathbf{F}_{\mathrm{X}}\right)$ represents the marginal increase in $R_{\mathrm{ip} 1, k}\left(\gamma_{k}\left(\mathbf{F}_{\mathrm{X}}\right)\right)$ per unit increase in $A_{k}\left(\mathbf{F}_{\mathrm{X}}\right)$. We can also interpret it as the desired signal reward at receiver $k$. After some manipulation, we obtain

$$
\begin{aligned}
& \theta_{\mathrm{BF}, \mathrm{ip} 1, k}\left(\mathbf{F}_{\mathrm{X}}\right) \\
& = \begin{cases}\frac{(1-t)}{\ln 2} \frac{\gamma_{k}^{2}\left(\mathbf{F}_{\mathrm{X}}\right)}{A_{k}\left(\mathbf{F}_{\mathrm{X}}\right)\left[1+\gamma_{k}\left(\mathbf{F}_{\mathrm{X}}\right)\right]}, & \text { if } \gamma_{k}\left(\mathbf{F}_{\mathrm{X}}\right) \leq \phi_{k}\left(\bar{\xi}_{k}, t\right) \\
\frac{(1-t)}{\ln 2} \frac{\gamma_{k}^{2}\left(\mathbf{F}_{\mathrm{X}}\right)\left[1+\phi_{k}\left(\bar{\xi}_{k}, t\right)\right]}{A_{k}\left(\mathbf{F}_{\mathrm{X}}\right)\left[1+\gamma_{k}\left(\mathbf{F}_{\mathrm{X}}\right)\right]^{2}}, & \text { otherwise, }\end{cases}
\end{aligned}
$$

$$
\beta_{\mathrm{BF}, \mathrm{ip} 1, k}\left(\mathbf{F}_{\mathrm{X}}\right)=\frac{\theta_{\mathrm{BF}, \mathrm{ip} 1, k}\left(\mathbf{F}_{\mathrm{X}}\right)}{\gamma_{k}\left(\mathbf{F}_{\mathrm{X}}\right)} .
$$

To compute $\theta_{\mathrm{BF}, \mathrm{ip} 1, k}\left(\mathbf{F}_{\mathrm{X}}\right)$, receiver $k$ itself estimates $\gamma_{k}\left(\mathbf{F}_{\mathrm{X}}\right)$ and $A_{k}\left(\mathbf{F}_{\mathbf{X}}\right)$. In addition, it obtains $\phi_{k}\left(\bar{\xi}_{k}, t\right)$ from relay $k$ via a feedforward channel. We need $\theta_{\mathrm{BF}, \mathrm{ip} 1, k}\left(\mathbf{F}_{\mathrm{X}}\right)$ and $\gamma_{k}\left(\mathbf{F}_{\mathrm{X}}\right)$ to compute $\beta_{\mathrm{BF}, \mathrm{ip} 1, k}\left(\mathbf{F}_{\mathrm{X}}\right)$.

We next present in detail how to use interference prices and desired signal rewards in the proposed relay beamforming design. Applying the chain rule, we can show that (21) is equivalent to

$\beta_{\mathrm{BF}, \mathrm{ip} 1, k}\left(\mathbf{F}_{\mathrm{X}}\right) \frac{\partial A_{k}\left(\mathbf{F}_{\mathrm{X}}\right)}{\partial \mathbf{f}_{\mathrm{X}, k}^{*}}-\sum_{m \neq k} \theta_{\mathrm{BF}, \mathrm{tip} 1, m}\left(\mathbf{F}_{\mathrm{X}}\right) \frac{\partial I_{k}\left(\mathbf{F}_{\mathrm{X}}\right)}{\partial \mathbf{f}_{\mathrm{X}, k}^{*}}=\lambda_{k} \mathbf{f}_{\mathrm{X}, k} \cdot$

Equivalently,

$$
\begin{aligned}
& {\left[\beta_{\mathrm{BF}, \mathrm{ip} 1, k}\left(\mathbf{F}_{\mathrm{X}}\right) \mathbf{h}_{k, k} \mathbf{h}_{k, k}^{*}-\sum_{m \neq k} \theta_{\mathrm{BF}, \mathrm{tip} 1, m}\left(\mathbf{F}_{\mathrm{X}}\right) \mathbf{h}_{m, k} \mathbf{h}_{m, k}^{*}\right]} \\
& \times \mathbf{x}_{\mathrm{XF}, k}\left(\mathbf{F}_{\mathrm{X}}\right)
\end{aligned}
$$

The proposed algorithm is iterative and asynchronous. In each iteration, only one relay $k$ is selected randomly to update its transmit beamformer by solving the following problem

$$
\left(\mathcal{B F}-\mathcal{N} \mathcal{P}_{k}\right): \max _{\mathbf{s} \in \mathbb{F}_{\mathrm{X}, k}} \mathbf{s}^{*} \mathbf{X}_{\mathrm{BF}, k}\left(\mathbf{s} ; \mathbf{F}_{\mathrm{X},-k}\right) \mathbf{s} .
$$

According to Proposition 3 in [35], the resulting algorithm based on $R_{\mathrm{ip} 1, k}\left(\gamma_{k}\left(\mathbf{F}_{\mathrm{X}}\right)\right)$ is guaranteed to converge to a stationary point of $(\mathcal{B F}-\mathcal{I P})$.

When the relays are equipped with multiple antennas, i.e., $N_{\mathrm{X}}>1$, in general, $\left(\mathcal{B \mathcal { F }}-\mathcal{N P}_{\boldsymbol{k}}\right)$ is a nonlinear optimization problem, and hence finding its globally optimal solutions may be time-consuming. To overcome this limitation, we adopt the method in [17] to propose a modified algorithm with a simple beamforming update rule. In particular, in each iteration $n \geq 1$, we propose to replace
$\mathbf{X}_{\mathrm{BF}, k}\left(\mathbf{F}_{\mathrm{X}}^{(n)}\right)$ by $\mathbf{Y}_{\mathrm{BF}, k}\left(\mathbf{F}_{\mathrm{X}}^{(n-1)}\right)$, which is defined as

$$
\begin{aligned}
\mathbf{Y}_{\mathrm{BF}, k}\left(\mathbf{F}_{\mathrm{X}}^{(n-1)}\right)= & \beta_{\mathrm{BF}, \mathrm{ip} 1, k}\left(\mathbf{f}_{\mathrm{X}, k}^{(n-1)} ; \mathbf{F}_{\mathrm{X},-k}^{(n-1)}\right) \mathbf{h}_{k, k} \mathbf{h}_{k, k}^{*} \\
& -\sum_{m \neq k} \theta_{\mathrm{BF}, \mathrm{ip} 1, m}\left(\mathbf{f}_{\mathrm{X}, m}^{(n-1)} ; \mathbf{F}_{\mathrm{X},-m}^{(n-1)}\right) \mathbf{h}_{m, k} \mathbf{h}_{m, k}^{*} .
\end{aligned}
$$

Both $\beta_{\mathrm{BF}, \mathrm{ip} 1, k}\left(\mathbf{f}_{\mathrm{X}, k}^{(n-1)} ; \mathbf{F}_{\mathrm{X},-k}^{(n-1)}\right)$ and $\theta_{\mathrm{BF}, \mathrm{ip} 1, m}\left(\mathbf{f}_{\mathrm{X}, m}^{(n-1)} ; \mathbf{F}_{\mathrm{X},-m}^{(n-1)}\right)$ for $m \neq k$ are computed in the previous iteration, therefore $\mathbf{Y}_{\mathrm{BF}, k}\left(\mathbf{F}_{\mathrm{X}}^{(n-1)}\right)$ is independent of $\mathbf{s}$. In iteration $n$, the selected relay $k$ needs to solve the following problem to determine $\mathbf{f}_{\mathrm{X}, k}^{(n)}$

$$
\left(\mathcal{B F}-\mathcal{A N} \mathcal{P}_{k}\right): \max _{\mathbf{s} \in \mathbb{F}_{\mathrm{X}, k}} \mathbf{s}^{*} \mathbf{Y}_{\mathrm{BF}, k}\left(\mathbf{F}_{\mathrm{X}}^{(n-1)}\right) \mathbf{s}
$$

We can show that the solution is $\mathbf{f}_{\mathrm{X}, k}^{(n)}=$ $v^{\max }\left(\mathbf{Y}_{\mathrm{BF}, k}\left(\mathbf{F}_{\mathrm{X}}^{(n-1)}\right)\right)$, the eigenvector corresponding to the maximum non-negative eigenvalue of $\mathbf{Y}_{\mathrm{BF}, k}\left(\mathbf{F}_{\mathrm{X}}^{(n-1)}\right)$. If $\mathbf{Y}_{\mathrm{BF}, k}\left(\mathbf{F}_{\mathrm{X}}^{(n-1)}\right)$ has no non-negative eigenvalue, then the relay does not update its beamforming.

Algorithm. Distributed two-hop interference pricing algorithm for relay beamforming design

- Initialization: each relay $k$ feeds forward $\phi_{k}\left(\bar{\xi}_{k}, t\right)$ to receiver $k$ and selects a random beamformer $\mathbf{f}_{\mathrm{X}, k}^{(0)}$.

- Iteration $n(n \geq 1)$ : the following updates are performed

- Interference price: each receiver $k$ computes $\theta_{\mathrm{BF}, \mathrm{ip} 1, k}\left(\mathbf{F}_{\mathrm{X}}^{(n-1)}\right)$ and broadcasts to all the relays.

- Desired signal reward: each receiver $k$ computes $\beta_{\mathrm{BF}, \mathrm{ip} 1, k}\left(\mathbf{F}_{\mathrm{X}}^{(n-1)}\right)$ and $\gamma_{k}\left(\mathbf{F}_{\mathrm{X}}^{(n-1)}\right)$. Then it feeds back the information to relay $k$.

- Beamforming vector: a relay $k$ is randomly selected to update $\mathbf{f}_{\mathrm{X}, k}^{(n)}=v^{\max }\left(\mathbf{Y}_{\mathrm{BF}, k}\left(\mathbf{F}_{\mathrm{X}}^{(n-1)}\right)\right)$.

Although we have not been able to prove analytically the convergence of the modified algorithm, we observed from our numerical results that it converges in all the cases considered.

For updating its beamforming vector, each relay $k$ needs to know the following information: $\left\{\mathbf{h}_{m, k}\right\}_{m=1}^{K}, \theta_{\mathrm{BF}, \mathrm{ip} 1, m}$ for $m \neq k$, and $\beta_{\mathrm{BF}, \mathrm{ip} 1, k}$. While this information can be made available at the relay via feedback, thus allowing for distributed implementation, a large amount of overhead may incur. In addition, in this article, we assume that the relays have obtained this information perfectly. The inaccuracy of this information, however, may affect significantly the achievable sum-rates. Methods for reducing overhead and investigating the impacts of the uncertainty of this 
information on the sum-rate performance are interesting topics for future research.

Note that our proposed algorithm is different from the prior algorithm in [16]. Prior work in [16] is able to maximize the sum-rates on a single hop, i.e., either the first hop or the second hop. It ignores $\left\{\gamma_{k}^{\{1\}}\right\}_{k=1}^{K}$ and $t$ and hence results in two-hop rate mismatch. On the contrary, our approach is able to take into account $\left\{\bar{\xi}_{k}\right\}_{k=1}^{K}$ and $t$ to alleviate two-hop rate mismatch to obtain higher end-toend sum-rates. In addition, the relays need to feedforward information to their associated receivers.

Recall that when $N_{\mathrm{X}}>1$, then $\left(\mathcal{B F}-\mathcal{N P}_{k}\right)$ is a nonlinear optimization problem, of which finding the globally optimal solutions may be time-consuming. Although the modified algorithm can approximately solve $\left(\mathcal{B F}-\mathcal{N} \mathcal{P}_{k}\right)$, we are unable to prove analytically the convergence of the modified algorithm. This challenge may not appear when we focus on updating only the actual transmit power $\mathbf{p}_{\mathrm{X}}$, for example, when the relays have a single antenna or when we update only the norm of the relay precoders but not their shape. For notational convenience, we denote $\mathbf{F}_{\mathrm{X}}=\sqrt{p_{\mathrm{X}}} \overline{\mathbf{F}}_{\mathrm{X}}$, where $\overline{\mathbf{F}}_{\mathrm{X}}$ with $\left\|\overline{\mathbf{F}}_{\mathrm{X}}\right\|_{F}^{2}=1$ represents the shape of the transmit precoders at the relays. Given fixed beamforming vectors $\overline{\mathbf{F}}_{\mathrm{X}}$, we define the effective channel from relay $k$ to receiver $m$ as $\bar{h}_{m, k} \triangleq \mathbf{h}_{m, k}^{*} \overline{\mathbf{f}}_{X, k} \in \mathbb{C}$ for $k, m \in \mathcal{K}$. We can always formulate an interference pricing-based relay power control problem to determine px given the knowledge of effective channel $\bar{h}_{m, k}$, which is referred to as $(\mathcal{P C}-\mathcal{I P})$ and is a special case of $(\mathcal{B F}-$ $\mathcal{I P})$. Note that in the relay beamforming design problem, each relay needs to determine simultaneously several complex numbers to update its beamforming vectors. In the relay power control algorithm, each relay needs to determine only a positive real number for its transmit power value. This may simplify the implementation of the resulting algorithm. Specifically, by relaxing the power range constraints and using similar steps for finding highquality solutions for $(\mathcal{B F}-\mathcal{I P})$, we can develop a two-hop interference pricing power control algorithm for determining $\mathbf{p}_{\mathrm{X}}$. This algorithm is iterative and asynchronous where in each iteration only one relay is allowed to update the transmit power. Let $k$ be the index of the selected relay for transmit power update in iteration $n \geq 1$. One important characteristic of this algorithm is that the candidate value for power update in iteration $n$ is provided in closed form as where

$$
\begin{aligned}
& \theta_{\mathrm{PC}, \mathrm{ip} 1, k}\left(\mathbf{p}_{\mathrm{X}}\right) \\
& =\left\{\begin{array}{l}
\frac{(1-t)}{\ln 2} \frac{\gamma_{k}^{2}\left(\overline{\mathbf{F}}_{\left.\mathrm{X}, \mathbf{p}_{\mathrm{X}}\right)}\right.}{p_{\mathrm{X}, k}\left|\bar{h}_{k, k}\right|^{2}\left[1+\gamma_{k}\left(\overline{\mathbf{F}}_{\mathrm{X}}, \mathbf{p}_{\mathrm{X}}\right)\right]}, \text { if } \gamma_{k}\left(\overline{\mathbf{F}}_{\mathrm{X}}, \mathbf{p}_{\mathrm{X}}\right) \leq \phi_{k}\left(\bar{\xi}_{k}, t\right) \\
\frac{(1-t)}{\ln 2} \frac{\left[1+\phi_{k}\left(\bar{\xi}_{k}, t\right)\right] \gamma_{k}^{2}\left(\overline{\mathbf{F}}_{\mathrm{X}}, \mathbf{p}_{\mathrm{X}}\right)}{p_{\mathrm{X}, k}\left|\bar{h}_{k, k}\right|^{2}\left[1+\gamma_{k}\left(\overline{\mathbf{F}}_{\mathrm{X}}, \mathbf{p}_{\mathrm{X}}\right)\right]^{2}}, \text { otherwise. }
\end{array}\right.
\end{aligned}
$$

Incorporating back the power range constraints, we propose that relay $k$ updates its transmit power as $p_{\mathrm{X}, k}^{(n)}=\bar{p}_{\mathrm{X}, k}^{(n)}$ only if $0 \leq \bar{p}_{\mathrm{X}, k}^{(n)} \leq p_{\mathrm{X}, k}^{\max }$. According to Proposition 2 in [35], the iterative and asynchronous interference pricing algorithm for power control is guaranteed to converge to a stationary point of $(\mathcal{P C}-\mathcal{I P})$. Note that this algorithm does not maximize directly the exact end-to-end sum-rates. Thus, its convergence in terms of exact endto-end sum-rate maximization is not guaranteed. In our simulations, however, this algorithm converges in all the considered scenarios.

\section{Discussion}

In this section, we provide some remarks on the order of optimization, distributed implementation, and complexity analysis of the algorithm.

\section{Order of optimization}

There is another optimization order where the relays are first designed to maximize the achievable sum-rates on the second hop. Then interference pricing is used to take into account the timesharing and second-hop rates in the design the transmitters to approximately maximize end-to-end achievable rates. Depending on channel realizations on two hops, one order of the optimization outperforms the other in terms of end-to-end sum-rate maximization and vice versa. We prefer our order of optimization due to overhead considerations. Specifically, as the relays themselves estimate the received SINR on the first hop, our proposed order of optimization only requires the receivers estimate and send back the secondhop SINR to the relays to perform two-hop rate matching. The other order of optimization, however, requires the relays and receivers to send back the SINR on two hops to the transmitters to perform two-hop rate-matching.

\section{Distributed implementation}

The implementation of the proposed algorithm requires that the transmissions on each hop start at the same

$$
\bar{p}_{\mathrm{X}, k}^{(n)}=\left\{\begin{array}{l}
{\left[\frac{(1-t)}{\ln 2} \frac{\gamma_{k}\left(\overline{\mathbf{F}}_{\mathrm{X}}, \mathbf{p}_{\mathrm{X}}^{(n-1)}\right)}{p_{\mathrm{X}, k}^{(n-1)}} \frac{1}{\sum_{m \neq k} \theta_{\mathrm{PC}, \mathrm{p} 1, m}\left(\mathbf{p}_{\mathrm{X}}^{(n-1)}\right)\left|\bar{h}_{m, k}\right|^{2}}-1\right] \frac{p_{\mathrm{X}, k}^{(n-1)}}{\gamma_{k}\left(\overline{\mathbf{F}}_{\mathrm{X}}, \mathbf{p}_{\mathrm{X}}^{(n-1)}\right)}, \text { if } \gamma_{k}\left(\overline{\mathbf{F}}_{\mathrm{X}}, \mathbf{p}_{\mathrm{X}}^{(n-1)}\right) \leq \phi_{k}\left(\bar{\xi}_{k}, t\right)} \\
{\left[\frac{(1-t)}{\ln 2} \frac{\gamma_{k}\left(\overline{\mathbf{F}}_{\mathrm{X}}, \mathbf{p}_{\mathrm{X}}^{(n-1)}\right)}{p_{\mathrm{X}, k}^{(n-1)}} \frac{\left[1+\phi_{k}\left(\bar{\xi}_{k}, t\right)\right]}{\sum_{m \neq k} \theta_{\mathrm{PC}, \mathrm{ip} 1, m}\left(\mathbf{p}_{\mathrm{X}}^{(n-1)}\right)\left|\bar{h}_{m, k}\right|^{2}}-1\right]^{1 / 2} \frac{p_{\mathrm{X}, k}^{(n-1)}}{\gamma_{k}\left(\overline{\mathbf{F}}_{\mathrm{X}}, \mathbf{p}_{\mathrm{X}}^{(n-1)}\right)}, \text { otherwise, }}
\end{array}\right.
$$


time and end at the same time. The relays are informed about the predetermined common time sharing value $t$. The transmitters need to agree with each other on the transmission strategy on the first hop as listed in Step 1. Each relay $k$ itself estimates the received SINR on the first hop $\bar{\xi}_{k}$ and computes $\phi_{k}\left(\bar{\xi}_{k}, t\right)$. Each relay $k$ feeds forward $\phi_{k}\left(\bar{\xi}_{k}, t\right)$ to receiver $k$ and selects a random beam former $\mathbf{f}_{\left.\mathrm{X}, k^{(}\right)}^{(0)}$. In each iteration $n \geq 1$, the following updates are performed in the predetermined order: (i) each receiver $k$ computes $\theta_{\mathrm{BF}, \mathrm{ip} 1, k}\left(\mathbf{F}_{\mathrm{X}}^{(n-1)}\right)$ and broadcasts it to all the relays, (ii) each receiver $k$ computes $\beta_{\mathrm{BF}, \mathrm{ip} 1, k}\left(\mathbf{F}_{\mathrm{X}}^{(n-1)}\right)$ and $\gamma_{k}\left(\mathbf{F}_{\mathrm{X}}^{(n-1)}\right)$ and then sends them back to relay $k$, and (iii) a relay $k_{0}$ is randomly selected to update its beamforming vector $\mathbf{f}_{\mathrm{X}, k_{0}}^{(n)}=v^{\max }\left(\mathbf{Y}_{\mathrm{BF}, k_{0}}\left(\mathbf{F}_{\mathrm{X}}^{(n-1)}\right)\right)$. Note that this implementation requires feedback and feedforward mechanisms between the relays and receivers. It also requires a little coordination among the relays to determine which relay is selected to update its beamforming vector in each iteration.

\section{Complexity analysis}

For simplicity in the complexity analysis of the algorithm, we assume that $N_{k}=N_{\mathrm{X}}$ for $k \in \mathcal{K}$. Note that the initialization of the algorithm requires the estimation of the received SINR on the first hop $\bar{\xi}_{k}$ and then the computation of $\phi_{k}\left(\bar{\xi}_{k}, t\right)$ based on (15). Moreover, this step is performed only once at the beginning. Thus, in the complexity analysis, we ignore the initialization and focus on the iterations. Recall that in each iteration, only one relay is allowed to update its beamformer. We now provide a rough complexity analysis of the individual steps in each iteration. First, in the interference price update step, we need to compute $I_{k}\left(\mathbf{F}_{\mathrm{X}}\right)=\sum_{q \neq k}\left|\mathbf{f}_{\mathrm{X}, q}^{*} \mathbf{h}_{k, q}\right|^{2}$ and $A_{k}\left(\mathbf{F}_{\mathrm{X}}\right)=$ $\left\|\mathbf{f}_{\mathrm{X}, k}^{*} \mathbf{h}_{k, k}\right\|^{2}$ for each $k \in \mathcal{K}$, which allow us to compute $\gamma_{k}\left(\mathbf{F}_{\mathrm{X}}\right)$ according to (7) and then $\theta_{\mathrm{BF}, \mathrm{ip} 1, k}\left(\mathbf{F}_{\mathrm{X}}\right)$ according to (25). Thus, this step yields a complexity of $\mathcal{O}\left(K^{2} N_{\mathrm{X}}\right)$. Second, in the desired signal reward update step, we need to compute $\beta_{\mathrm{BF}, \mathrm{ip} 1, k}\left(\mathbf{F}_{\mathrm{X}}\right)$ from $\theta_{\mathrm{BF}, \mathrm{ip} 1, k}\left(\mathbf{F}_{\mathrm{X}}\right)$ and $\gamma_{k}\left(\mathbf{F}_{\mathrm{X}}\right)$ according to (26). This step can be ignored from the complexity analysis. Third, in the beamforming vector update step, we need to compute $\mathbf{Y}_{\mathrm{BF}, k}\left(\mathbf{F}_{\mathrm{X}}^{(n-1)}\right)$ according to (30) only for the selected relay $k$. This computation has the complexity of $\mathcal{O}\left(K N_{\mathrm{X}}^{2}\right)$. We also need to perform an eigenvalue decomposition of $\mathbf{Y}_{\mathrm{BF}, k}\left(\mathbf{F}_{\mathrm{X}}^{(n-1)} \in \mathbb{C}^{N_{\mathrm{X}} \times N_{\mathrm{X}}}\right.$, which yields a complexity of $\mathcal{O}\left(N_{\mathrm{X}}^{3}\right)$. Thus, this step has a complexity of $\mathcal{O}\left(K N_{\mathrm{X}}^{2}\right)+\mathcal{O}\left(N_{\mathrm{X}}^{3}\right)$. In short, the per-iteration complexity of the algorithm is $\mathcal{O}\left(K^{2} N_{\mathrm{X}}\right)+\mathcal{O}\left(K N_{\mathrm{X}}^{2}\right)+$ $\mathcal{O}\left(N_{\mathrm{X}}^{3}\right)$.

\section{Numerical results}

This section provides Monte Carlo simulation results to evaluate the two-hop sum-rate performance of the proposed algorithms in a multicell cellular network setting.

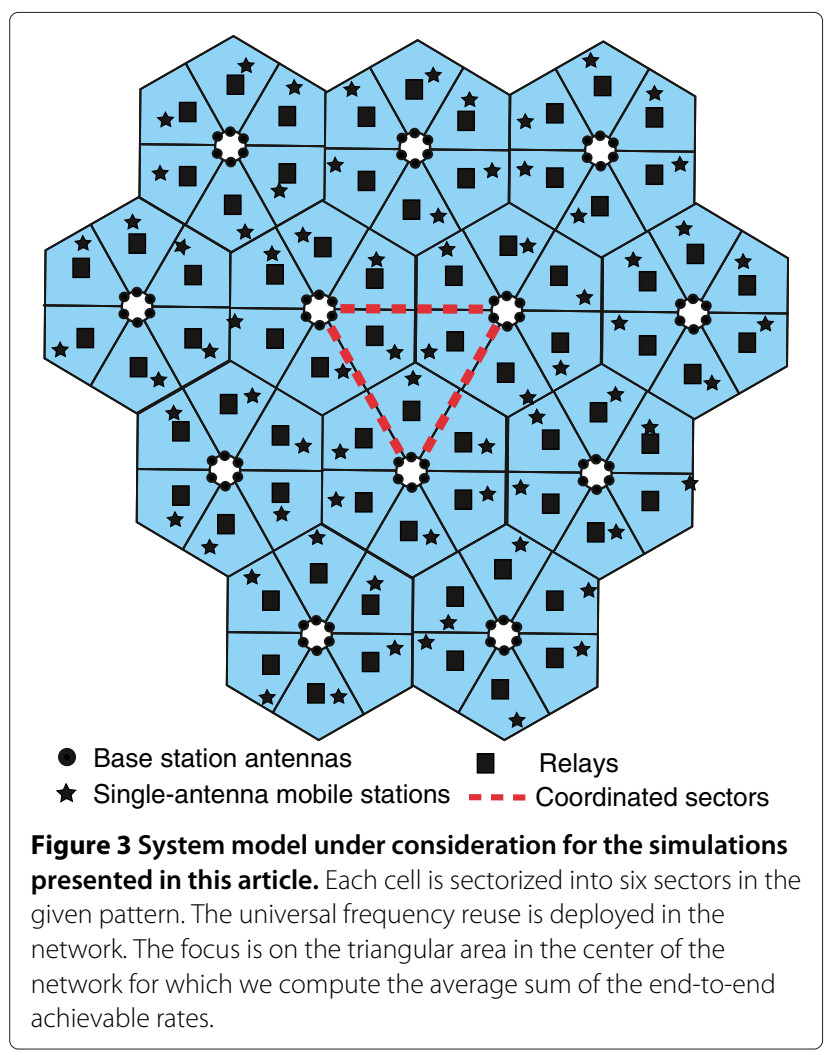

Universal frequency reuse is deployed in the network. The cells are sectorized such that each cell consists of six sectors as shown in Figure 3. The cell radius is $r=866 \mathrm{~m}$. More details in the simulation setting such as channel model parameters are referred to [6] to save space. The three sectors of interest are in the marked triangular area in the center of the network. Treating the interference from the other sectors than the ones of interest as additive

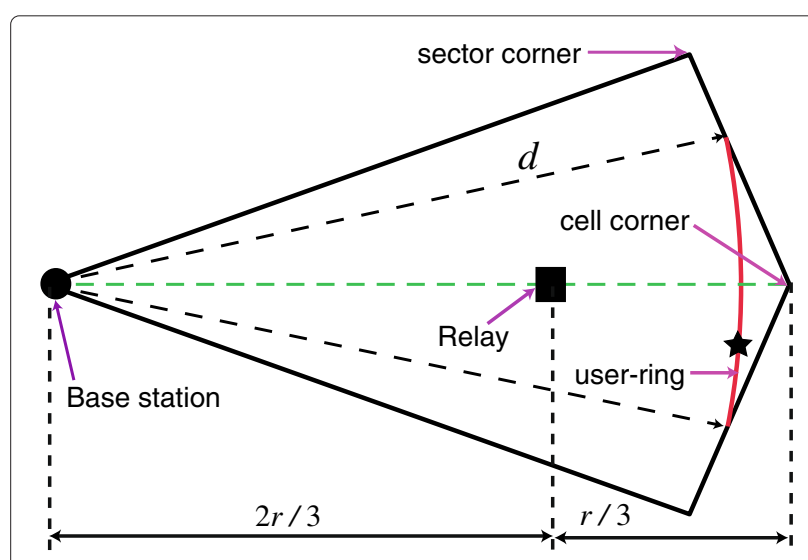

Figure 4 Illustration of user generation in the sectors of interest. Users are generated randomly on rings centered at their corresponding base stations. 


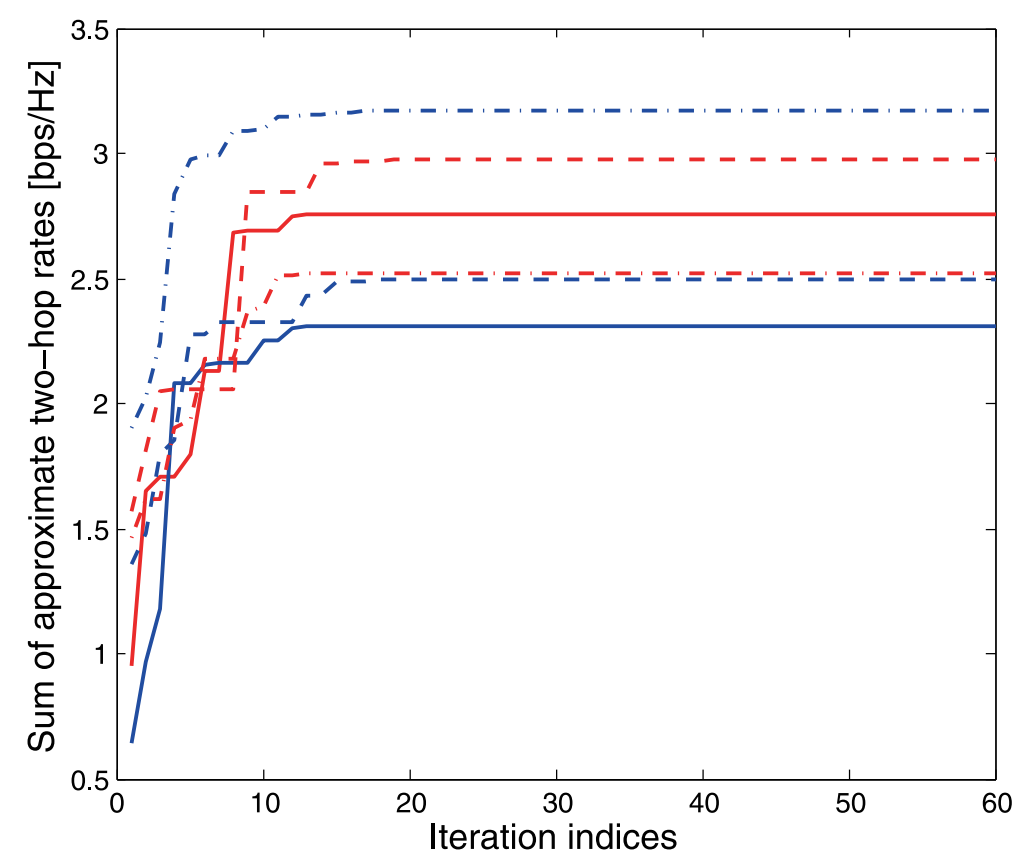

Figure 5 Investigation of the convergence of the proposed distributed relay beamforming algorithm. The proposed two-hop interference pricing beamforming converges well in terms of maximizing sum of the approximate end-to-end achievable rates.

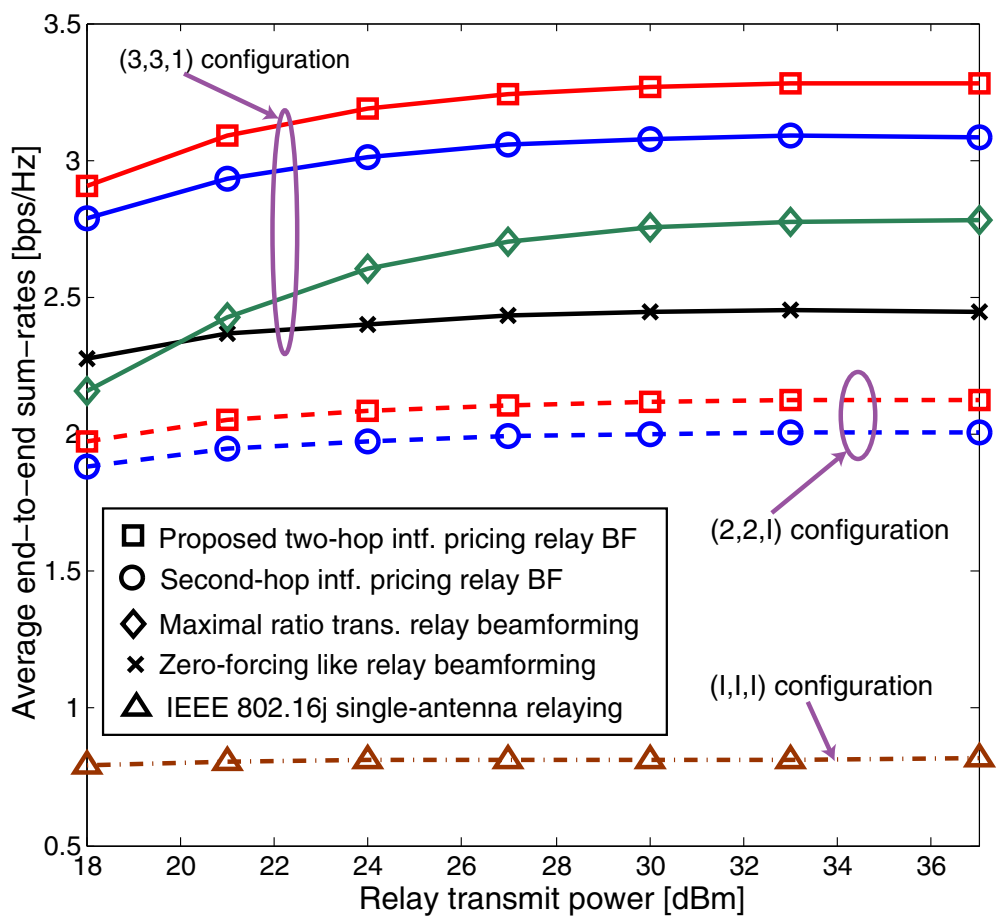

Figure 6 Sum-rate performance of relay beamforming algorithms for cell-edge users as functions of relay transmit power. We use $t=0.45 .(M, N, 1)$ means that each base station has $M$ antennas and each relay has $N$ antennas. 
Gaussian noise, we compute the average two-hop sumrates of the three pairs in the sectors of interest. The base stations use MRT beamforming to send data to their associated relays.

Figure 4 illustrates how users are generated in a sector and the location of the fixed relay. The distance from the relay in each sector to the base station is equal to two-third of the cell radius. The users in each sector of interest are generated randomly on the rings centered at their corresponding base station with radius $d$. Users in other sectors are generated randomly. We consider different antenna configurations, denoted as $(M, N, 1)$, i.e., $M$ directional antennas in each sector at a base station are used to serve a single user at a time via a dedicated relay with $N$ omnidirectional antennas. All relays have a common value of maximum transmit power $P^{\text {max }}$. We consider two sets of experiments: (i) varying relay power and (ii) moving users. In the first set of experiments, we assume that the users of interest moving on rings with $d=826 \mathrm{~m}$ are considered as cell edge users. The resulting plots show the average two-hop sum-rates as functions of $P^{\max }$. In the second set of experiments, we plot the average two-hop sum-rates as functions of $d$ when all the relays have $P^{\max }=37 \mathrm{dBm}$.

For comparison, we implement several baseline relay beamforming algorithms. While the ZF-like beamforming minimizes the sum of interference power each relay cause to the other receivers of interest, the MRT beamforming maximizes the desired signal power each relay sends to its associated receiver. We also implement the beamforming algorithm resulting from the direct application of single-hop interference pricing beamforming in [16]

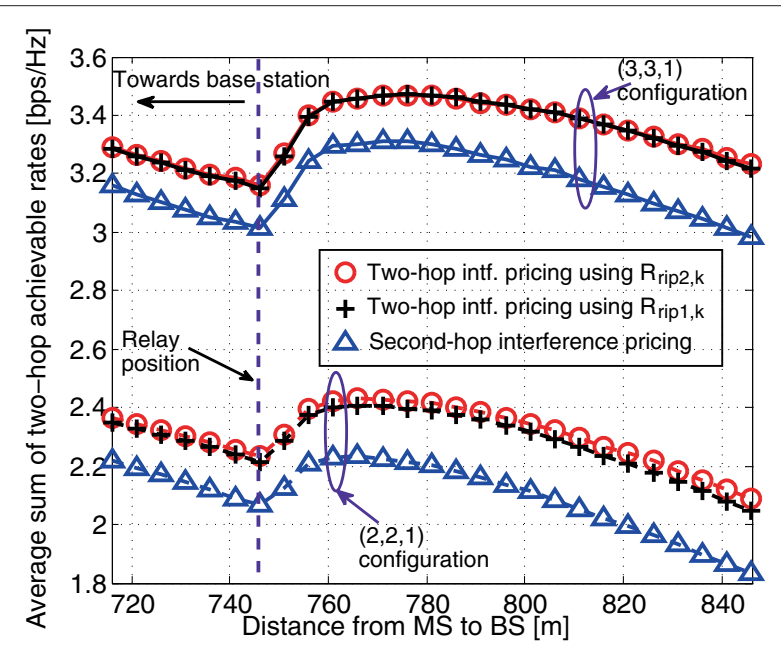

Figure 7 End-to-end achievable rates as functions of the distance from users' location to the base stations for $(3,3,1)$ and $(2,2,1)$ configurations. The algorithm based on $R_{\mathrm{ip} 2, k}\left(\mathbf{F}_{\mathrm{X}}\right)$ slightly outperforms the algorithm based on $R_{\mathrm{ip} 1, k}\left(\mathbf{F}_{\mathrm{X}}\right)$ in terms of maximizing the exact end-to-end sum-rates.

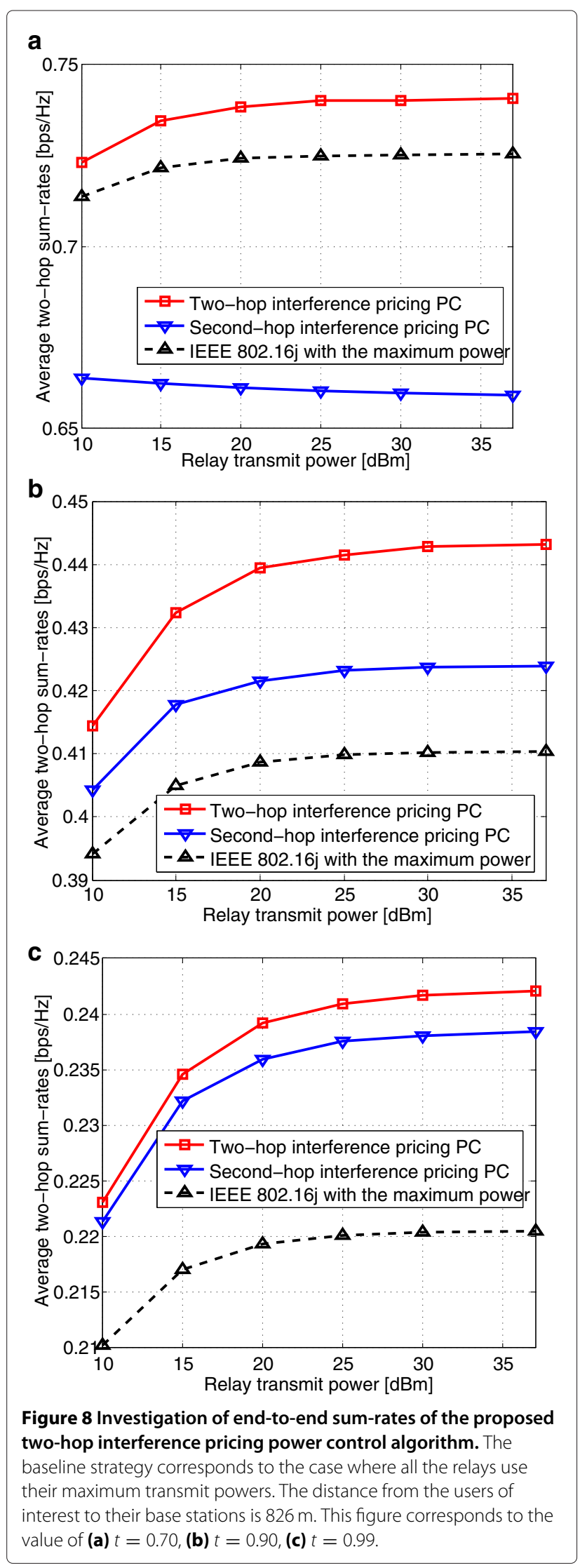


to the second-hop, which we refer to as the second-hop interference pricing beamforming.

We now investigate the convergence of the proposed distributed relay beamforming algorithm. Figure 5 shows the sum of the approximate end-to-end rates over iterations for the proposed two-hop interference pricing beamforming with the approximate rate function $R_{\mathrm{ip} 2, k}\left(\gamma_{k}\left(\mathbf{F}_{\mathrm{X}}\right)\right)$ given in (17). We notice that the approximate end-to-end sum-rates are not decreasing over iterations of the proposed relay beamforming algorithm. Moreover, the proposed algorithm converges quite fast and saturates within 20 iterations.

Figure 6 presents simulation results for different antenna configurations. First, we notice that the proposed algorithm outperforms the second-hop interference pricing algorithm in both cases: $5.8 \%$ gain in the $(2,2,1)$ case and $6.4 \%$ gain in the $(3,3,1)$ case. These gains come from the consideration of the first-hop performance and the timesharing value, which alleviates rate mismatch. Note that our proposed algorithm requires only additional overhead for feed-forwarding $\phi_{k}\left(\bar{\xi}_{k}, t\right)$ from each relay $k$ to receiver $k$ in the initial step. Since the channels between base stations and relays are expected to be stable, such additional overhead is negligible. Second, for the $(3,3,1)$ configuration, the two-hop interference pricing relay beamforming provides $34 \%$ gain over the MRT beamforming and $18 \%$ gain over the ZF-like beamforming. Finally, the proposed algorithm provides large gain over IEEE 802.16j single-antenna relay communication: $160 \%$ in the $(2,2,1)$ case and $302 \%$ in the $(3,3,1)$ case. This highlights the benefits of multiple-antenna relays.

Figure 7 shows the simulation results of a moving user experiment for the two-hop interference pricing beamforming design algorithms using the two examples of approximate end-to-end rates as compared to the second-hop interference pricing. We notice that the twohop interference pricing algorithms provide large gains over the second-hop interference pricing for all range of user locations. Moreover, the two-hop interference pricing beamforming design with $R_{\mathrm{ip} 2, k}\left(\gamma_{k}\left(\mathbf{F}_{\mathrm{X}}\right)\right.$ always slightly outperforms that with $R_{\mathrm{ip} 1, k}\left(\gamma_{k}\left(\mathbf{F}_{\mathrm{X}}\right)\right.$ as expected. This is because $R_{\mathrm{ip} 2, k}\left(\gamma_{k}\left(\mathbf{F}_{\mathrm{X}}\right)\right.$ provides a better approximation of the end-to-end rate than does $R_{\mathrm{ip} 1, k}\left(\gamma_{k}\left(\mathbf{F}_{\mathrm{X}}\right)\right.$. This means the quality of the resulting solutions by the proposed twohop interference pricing beamforming design depends on the selection of the approximate end-to-end rate function. The same trend holds for other antenna cases although the simulation results are not shown here to save space.

The last experiment is on power control for the singleantenna relay interference channel with $M=N=1$. For comparison, we implement two reference strategies: (i) second-hop interference pricing algorithm and (ii) IEEE $802.16 \mathrm{j}$ with the maximum power. In the second-hop interference pricing algorithm, we apply directly the prior interference pricing algorithm for the single-hop interference channel in [15] for the second hop. In the maximumpower IEEE 802.16j strategy, the relays behave egoistically by using their maximum power to increase the desired signal power to their associated receivers while causing large interference to unintended receivers. Figure 8 provides the results for $d=836 \mathrm{~m}$ (cell-edge user case) and $t \in$ $\{0.70,0.90,0.95\}$. Note that the second-hop interference pricing algorithm may perform worse than the maximumpower IEEE 802.16j strategy. This confirms that the maximization of the second-hop sum-rates may cause more mismatch between the rates on two hops, degrading the two-hop sum-rate performance. In addition, our proposed two-hop interference pricing algorithm always outperforms the other algorithms for all considered values of $t$. The reason is that our proposed algorithm can take into account $t$ and $\left\{\bar{\xi}_{k}\right\}_{k=1}^{K}$ into the relay power control to alleviate two-hop rate mismatch while the other algorithms cannot.

\section{Conclusions}

We proposed an algorithm for designing the transmission parameters at the transmitters and relays in the DF relay interference channel to maximize end-to-end sum-rates. The algorithm copes with both interference and mismatch between the rates on two hops, two main challenges in designing the DF relay systems. Our key contribution is the two-hop interference pricing framework that allows for the integration of information about the first-hop performance and timesharing value in the computation of interference prices on the second hop for design the relays. The proposed algorithm allows for distributed implementation, making it suitable for practical systems. Simulations showed that the proposed algorithm obtains higher end-to-end sum-rates than the existing strategies, including the naïve approach of applying independently the single-hop interference pricing algorithms on two hops.

\section{Competing interests}

$\mathrm{RH}$ is also President and CEO of MIMO Wireless Inc. The terms of this arrangement have been reviewed and approved by the University of Texas at Austin in accordance with its policy on objectivity in research.

\section{Acknowledgements}

This study was supported by a grant from Huawei Technologies, Inc.

Received: 21 June 2012 Accepted: 11 January 2013

Published: 20 February 2013

\section{References}

1. O Simeone, O Somekh, Y Bar-Ness, HV Poor, S Shamai, in Proceedings of the Allerton Conference on Communication, Control, Computing. Capacity of linear two-hop mesh networks with rate splitting, decode-and-forward relaying and cooperation, (Monticello, IL, 2007)

2. PSC Thejaswi, A Bennatan, J Zhang, R Calderbank, D Cochran, in Proceedings of the Allerton Conference on Communication Control Computing. Rate-achievability strategies for two-hop interference flows, (Montecillo, IL, 2008), pp. 1432-1439 
3. Y Cao, B Chen, in Proceedings of the Allerton Conference on Communication, Control, Computing. Capacity bounds for two-hop interference networks, (Montecillo, IL, 2009), pp. 272-279

4. Y Shi, J Wang, K Letaief, R Mallik, A game-theoretic approach for distributed power control in interference relay channels. IEEE Trans. Wirel. Commun. 8(6), 3151-3161 (2009)

5. AMA Ahmed, I Marsland, in Proceedings of the IEEE International Conference on Communication. Co-channel interference cancellation in multihop relay networks, (Beijing, China, 2008), pp. 62-67

6. SW Peters, AY Panah, KT Truong, RWJr Heath, Relay architectures for 3GPP LTE-Advanced. EURASIP J. Wirel. Commun. Netw. 2009(618787), $1-14$ (2009)

7. H Ning, C Ling, KK Leung, in Proceedings of the IEEE International Symposium on Information Theory. Relay-aided interference alignment: feasibility conditions and algorithm, (Austin, TX, 2010), pp. 390-394

8. B Nourani, SA Motahari, AK Khandani, in Proceedings of the IEEE International Symposium on Information Theory. Relay-aided interference alignment for the quasi-static interference channel, (Austin, TX, 2010), pp. 405-409

9. T Gou, SA Jafar, C Wang, SW Jeon, SY Chung, Aligned interference neutralization and degrees of freedom of the $2 \times 2 \times 2$ interference channel. IEEE Trans. Inf. Theory. 58(7), 4381-4395 (2012)

10. E Yilmaz, F Boccardi, A Alexiou, in Proceedings of the IEEE Vehicular Technology Conference. Distributed and centralized architectures for relay-aided cellular systems, (Barcelona, Spain, 2009), pp. 1-5

11. IEEE 802.16j: Standard for local and metropolitan area networks_-Part 16: air interface for broadband wireless access systems_amendment 1 : Multiple relay specification

12. IEEE 802.16m Broadband Wireless Access Working Group: IEEE $802.16 \mathrm{~m}$ system requirement

13. 3GPP TR 36.814: Further enhancements for E-UTRA physical layer aspects

14. ZQ Luo, S Zhang, Dynamic spectrum management: complexity and duality. IEEE J. Sel. Topics Signal Process. 2, 57-73 (2008)

15. J Huang, RA Berry, ML Honig, Distributed interference compensation for wireless networks. IEEE J. Sel. Areas Commun. 24(5), 1074-1084 (2006)

16. C Shi, RA Berry, ML Honig, in Proceedings of the Allerton Conference on Communication, Control, Computing. Distributed interference pricing with MISO channels, (Monticello, IL, 2008), pp. 539-546

17. C Shi, RA Berry, ML Honig, in Proceedings of the IEEE Military Communications Conference. Local interference pricing for distributed beamforming in MIMO networks (Boston, MA, 2009), pp. 1-6

18. F Rashid-Farrokhi, KJR Liu, L Tassiulas, Transmit beamforming and power control for cellular wireless systems. IEEE J. Sel. Areas Commun. 16(8), 1437-1450 (1998)

19. G Scutari, D Palomar, S Barbarossa, Competitive design of multiuser MIMO systems based on game theory: a unified view. IEEE J. Sel. Areas Commun. 26(7), 1089-1103 (2008)

20. E Larsson, E Jorswieck, Competition versus cooperation on the MISO interference channel. IEEE J. Sel. Areas Commun. 26(7), 1059-1069 (2008)

21. SW Peters, RWJr Heath, Cooperative algorithms for MIMO interference channels. IEEE Trans. Veh. Technol. 60, 206-218 (2011)

22. D Schmidt, C Shi, RA Berry, ML Honig, W Utschick, Distributed resource allocation schemes. IEEE Signal Process. Mag. 26(5), 53-63 (2009)

23. R Zakhour, ZKM Ho, D Gesbert, in Proceedings of the IEEE Vehicular Technology Conference. Distributed beamforming coordination in multicell MIMO channels, (Barcelona, Spain, 2009), pp. 1-5

24. HChen, AB Gershman, S Shahbazpanahi, in Proceedings of the IEEE International Conference on Acoustics, Speech Signal Processing. Distributed peer-to-peer beamforming for multiuser relay networks (Taipei, Taiwan, 2009), pp. 2265-2268

25. DHN Nguyen, HH Nguyen, TT Pham, in Proceedings of the IEEE Global Telecommunication Conference. Distributed beamforming in multiuser multi-relay networks with guaranteed QoS, (Honolulu, HI, 2009), pp. 1-6

26. J Joung, AH Sayed, Multiuser two-way amplify-and-forward relay processing and power control methods for beamforming systems. IEEE Trans. Signal Process. 58(3), 1833-1846 (2010)

27. SW Jeon, SY Chung, SA Jafar, in Proceedings of the Allerton Conference on Communication, Control, Computing. Degrees of freedom of multi-source relay networks, (Monticello, IL, 2009), pp. 388-393
28. KT Truong, RWJr Heath, in Proceedings of the IEEE Asilomar Conference on Signals, Systems Computers. Interference alignment for multiple-antenna amplify-and-forward relay interference channel, (Pacific Grove, CA, USA, 2011), pp. 1-5

29. KT Truong, P Sartori, RWJr Heath, Cooperative algorithms for MIMO amplify-and-forward relay networks. IEEE Trans. Signal Process. 61(5), 1272-1287 (2013). [http://arxiv.org/abs/1112.4553] (to appear)

30. O Sahin, E Bala, R Yang, P Pietraski, in Proceedings of the IEEE Personal Indoor Mobile Radio Communication. Relaying with distributed interference alignment in multi-node networks, (Istanbul, Turkey, 2010), pp. 543-547

31. V Havary-Nassab, S Shahbazpanahi, A Grami, Optimal distributed beamforming for two-way relay networks. IEEE Trans. Signal Process. 58(3), 1238-1250 (2010)

32. CS Patel, GL Stuber, Channel estimation for amplify and forward relay based cooperation diversity systems. IEEE Trans. Wirel. Commun. 6(6), 2348-2356 (2007)

33. F Gao, T Cui, A Nallanathan, On channel estimation and optimal training design for amplify and forward relay networks. IEEE Trans. Wirel. Commun. 7(5), 1907-1916 (2008)

34. M Chiang, P Hande, T Lian, CW Tan, Power Control in Wireless Cellular Communications. Foundations and Trends in Communications and Information Theory. (Now Publishers Inc, Norwell, MA, 2008)

35. C Shi, RA Berry, ML Honig, in Proceedings of the IEEE International Symposium on Information Theory. Monotonic convergence of distributed interference pricing in wireless networks, (Seoul, Korea, 2009), pp. 1619-1623

36. GJ Foschini, Z Miljanic, A simple distributed autonomous power control algorithm and its convergence. IEEE Trans. Veh. Technol.

42(4), 641-646 (1993)

37. RD Yates, A framework for uplink power control in cellular radio systems IEEE J. Sel. Areas. Commun. 13(7), 1341-1347 (1995)

38. CU Saraydar, NB Mandayam, DJ Goodman, Efficient power control via pricing in wireless data networks. IEEE Trans. Commun. 50(2), 291-303 (2002)

39. CW Sung, WS Wong, A noncooperative power control game for multirate CDMA data networks. IEEE Trans. Wirel. Commun. 2, 186-194 (2003)

40. S Stanczak, M Wiczanowski, H Boche, Distributed utility-based power control: objectives and algorithms. IEEE Trans. Signal Process. 55(10), 5058-5068 (2007)

41. Y Su, M van Der Schaar, A new perspective on multi-user power control games in interference channels. IEEE Trans. Wirel. Commun. 8(6), 2910-2919 (2009)

42. LP Qian, YJ Zhang, J Huang, MAPEL: achieving global optimality for a non-convex wireless power control problem. IEEE Trans. Wirel. Commun. 8(3), 1553-1563 (2009)

43. S Stanczak, M Wiczanowski, H Boche, Fundamentals of Resource Allocation in Wireless Networks, 2nd edn. (Springer, New York, 2009)

44. V Chandrasekhar, JG Andrews, T Muharemovic, Z Shen, A Gatherer, Power control in two-tier femtocell networks. IEEE Trans. Wirel. Commun. 8(8), 4316-4328 (2009)

45. HC Jo, C Mun, J Moon, JG Yook, Interference mitigation using uplink power control for two-tier femtocell networks. IEEE Trans. Wirel. Commun. 8(10), 4906-4910 (2009)

46. S Zhou, H Xiao, Z Niu, in Proceedings of the IEEE International Conference on Communication. Distributed power control for interference-limited cooperative relay networks, (Dresden, Germany, 2009), pp. 1-6

47. Y Shi, RK Mallik, KB Letaief, in Proceedings of the IEEE International Conference on Communication. Power control for relay-assisted wireless systems with general relaying, (Cape Town, South Africa, 2010), pp. 1-5

48. L Xiao, L Cuthbert, in Proceedings of the IEEE Vehicular Technical Conference. Multi-cell non-cooperative power allocation game in relay based OFDMA systems, (Barcelona, Spain, 2009), pp. 1-5

49. KTruong, RWJr Heath, in Proceedings of the IEEE Global Telecommunication Conference. Relay beamforming using interference pricing for the two-hop interference channel, (Houston, TX, 2011), pp. 1-5

50. KT Truong, RWJr Heath, Joint transmit precoding for the relay interference broadcast channel. IEEE Trans. Veh. Technol. 62(3), 1201-1215 (2013). available at [http://arxiv.org/abs/1206.4275] (to appear)

51. A Chakrabarti, A Sabharwal, B Aazhang, in Proceedings of the IEEE Signal Processing Advanced Wireless Communication. Sensitivity of achievable rates for half-duplex relay channel, (New York City, NY, 2005), pp. 970-974 
52. YF Liu, YH Dai, ZQ Luo, in Proceedings of the IEEE International Conference on Acoustics, Speech Signal Processing. On the complexity of optimal coordinated downlink beamforming, (Dallas, TX, 2010)

53. EA Jorswieck, EG Larsson, D Danev, Complete characterization of the Pareto boundary for the MISO interference channel. IEEE Trans. Signal Process. 56(10), 5292-5296 (2008)

54. K Ko, CL Lin, Minimax and Applications chap. On the Complexity of Min-Max Optimization Problems and Their Approximation, (Kluwer, 1995), pp. 219-239

55. TKY Lo, Maximum ratio transmission. IEEE Trans. Commun. 47(10), 1458-1461 (1999)

56. ST Chung, SJ Kim, J Lee, JM Cioffi, in Proceedings of the IEEE International Symposium on Information Theory. A game-theoretic approach to power allocation in frequency-selective Gaussian interference channels, (Yokohama, Japan, 2003), p. 316

57. K Gomadam, VR Cadambe, SA Jafar, in Proceedings of the IEEE Global Telecommunication Conference. Approaching the capacity of wireless networks through distributed interference alignment (New Orleans, LA, 2008), pp. 1-6

58. SW Peters, RWJr Heath, in Proceedings of the IEEE International Conference on Acoustics, Speech Signal Processing. Interference alignment via alternating minimization, (Taipei, Taiwan, 2009), pp. 2445-2448

59. M Sadek, A Tarighat, AH Sayed, A leakage-based precoding scheme for downlink multi-user MIMO channels. IEEE Trans. Wirel. Commun. 6(5), 1711-1721 (2007)

60. SJ Kim, GB Giannakis, Optimal resource allocation for MIMO ad hoc cognitive radio networks. IEEE Trans. Inf. Theory. 57(5), 3117-3131 (2011)

61. Q Shi, M Razaviyayn, ZQ Luo, C He, An iteratively weighted MMSE approach to distributed sum-utility maximization for a MIMO interfering broadcast channel. IEEE Trans. Signal Process. 59(9), 4331-4340 (2011)

62. DP Bertsekas, Nonlinear Programming, 2nd edn. (Athena Scientific, Belmont, MA, 1999)

63. A Mas-Colell, MD Whinston, JR Green, Microeconomic Theory. (Oxford University Press, Oxford, UK, 1995)

doi:10.1186/1687-6180-2013-26

Cite this article as: Truong and Heath: A distributed algorithm using interference pricing for relay interference channels. EURASIP Journal on Advances in Signal Processing 2013 2013:26.

\section{Submit your manuscript to a SpringerOpen ${ }^{\circ}$ journal and benefit from:}

- Convenient online submission

- Rigorous peer review

- Immediate publication on acceptance

- Open access: articles freely available online

- High visibility within the field

- Retaining the copyright to your article

Submit your next manuscript at $\boldsymbol{p}$ springeropen.com 